\title{
Melanocortin-4 receptor antagonist TCMCB07 ameliorates cancer- and chronic kidney disease-associated cachexia
}

\author{
Xinxia Zhu, ${ }^{1}$ Michael F. Callahan, ${ }^{2}$ Kenneth A. Gruber, ${ }^{2,3,4}$ Marek Szumowski, ${ }^{1}$ and Daniel L. Marks ${ }^{1,5,6}$ \\ 'Papé Family Pediatric Research Institute, Oregon Health and Science University, Portland, Oregon, USA. ${ }^{2}$ Tensive Controls Inc., MU Life Sciences Business Incubator at Monsanto Place, Columbia, Missouri, \\ USA. ${ }^{3}$ Dalton Cardiovascular Research Center and ${ }^{4}$ Department of Medical Pharmacology and Physiology, University of Missouri, Columbia, Missouri, USA. ${ }^{5}$ Knight Cancer Institute and ${ }^{6}$ Brenden-Colson \\ Center for Pancreatic Care, Oregon Health \& Science University, Portland, Oregon, USA.
}

\begin{abstract}
Cachexia, a devastating wasting syndrome characterized by severe weight loss with specific losses of muscle and adipose tissue, is driven by reduced food intake, increased energy expenditure, excess catabolism, and inflammation. Cachexia is associated with poor prognosis and high mortality and frequently occurs in patients with cancer, chronic kidney disease, infection, and many other illnesses. There is no effective treatment for this condition. Hypothalamic melanocortins have a potent and long-lasting inhibitory effect on feeding and anabolism, and pathophysiological processes increase melanocortin signaling tone, leading to anorexia, metabolic changes, and eventual cachexia. We used 3 rat models of anorexia and cachexia (LPS, methylcholanthrene sarcoma, and 5/6 subtotal nephrectomy) to evaluate efficacy of TCMCB07, a synthetic antagonist of the melanocortin-4 receptor. Our data show that peripheral treatment using TCMCB07 with intraperitoneal, subcutaneous, and oral administration increased food intake and body weight and preserved fat mass and lean mass during cachexia and LPS-induced anorexia. Furthermore, administration of TСМСB07 diminished hypothalamic inflammatory gene expression in cancer cachexia. These results suggest that peripheral TCMCB07 treatment effectively inhibits central melanocortin signaling and therefore stimulates appetite and enhances anabolism, indicating that TCMCB07 is a promising drug candidate for treating cachexia.
\end{abstract}

\section{Introduction}

Cachexia is a devastating and multifactorial wasting syndrome, consisting of anorexia, loss of adipose tissue and lean body mass, and a paradoxical increase in energy expenditure and catabolism that accompanies a variety of illness conditions, such as cancer, chronic kidney disease (CKD), sepsis, chronic obstructive pulmonary disease, congestive heart failure, and HIV infection $(1,2)$. The severity of cachexia in these illnesses is often the primary determining factor for both quality of life and eventual mortality $(2,3)$. Cancer cachexia affects $50 \%$ to $80 \%$ of cancer patients, causes $20 \%$ to $40 \%$ of all cancer deaths, and aggravates toxicity and complications of cancer therapy (4-6). Advanced CKD, particularly with uremia, is frequently associated with cachexia. Survival with end-stage renal disease is even worse than with most cancers, and the mortality rate of maintenance dialysis patients is above $20 \%$ per year (7). At the patient level, longevity has consistently been observed in patients with CKD who have more muscle and/or fat, report better appetite, and eat more (1).

It has been known for 30 years and well established that the hypothalamic melanocortin system plays a central role in regulation of appetite, body mass, and energy homeostasis $(8,9)$.

Conflict of interest: MFC, KAG, and DLM have equity in Tensive Controls Inc. KAC and MFC were salaried officers of Tensive Controls Inc. DLM was the recipient of a subaward from KAG's NIH Small Business Innovation Research (SBIR) grant 2R44CA150703

Copyright: (C) 2020, American Society for Clinical Investigation.

Submitted: March 25, 2020; Accepted: June 10, 2020; Published: August 10, 2020

Reference information: J Clin Invest. 2020;130(9):4921-4934.

https://doi.org/10.1172/JCl138392.
Proopiomelanocortin- (POMC) and agouti-related peptide-expressing (AgRP-expressing) neurons located in the arcuate nucleus of the hypothalamus are the primary regulators of melanocortin signaling in the brain. This system is unique, not only in having the capability of sensing signals from a wide array of hormones, nutrients, and afferent neural inputs, but also in having the ability of transducing both anorexigenic agonists (e.g., $\alpha$-melanocyte-stimulating hormone $[\alpha-\mathrm{MSH}])$ and orexigenic antagonists/inverse agonists (e.g., AgRP) of melanocortin-3 and melanocortin-4 receptors (MC3R and MC4R). While MC3R neurons likely contribute to behavioral adaptation to fasting and nutrient partitioning, MC4R neurons are involved in feeding behavior, adaptive thermogenesis, and glucose homeostasis (10). Therefore, this system provides a logical target for developing drugs for treating cachexia, obesity, and diabetes $(8,11-14)$. The pathophysiological processes of many illnesses increase the melanocortin tone that suppresses appetite and anabolism, leading to anorexia and body weight loss, with inflammation as an essential driver (15). Inflammatory signals produced from acute illness responses and chronic conditions exert great influence on the hypothalamus perturbing the homeostatic system (16-18). Direct experimental evidence demonstrates that stimulating the hypothalamus with inflammatory cytokines, such as IL-1, IL-6, TNF- $\alpha$, and leukemia inhibitory factor, leads to anorexia via altering the activity of POMC and AgRP neurons (1922). Furthermore, increasing evidence supports that pathogenesis of cachexia caused by cancer, CKD, and many other chronic illnesses is tightly linked to inflammation (1, 6, 23-29).

Due to the complexity of pathogenesis and multifactorial pathophysiology of cachexia, and despite increased understanding of the mechanisms and many years of drug development 
effort, currently no effective medical intervention completely reverses cachexia and there are no approved drug therapies (5). A few potential treatments have been reported, including ghrelin receptor agonists and leptin antagonists (30-36), but these have not yet gained approval for treatment of patients with cachexia. The recent consensus and strategy in cachexia therapy is that adequate nutritional support remains a mainstay, whereas it is important to develop drugs that target overactivation of inflammation, catabolic processes, and cell injury $(5,6)$. Melanocortin antagonists, as powerful orexigenic agents in simulation of appetite, have been investigated for more than a decade (37-39). The efficacy of several compounds was evaluated in animal models, including our previous preclinical trials (40-42). However, to date, there are no drugs in this class that are approved for clinical treatment, highlighting the need to develop novel drugs with maximum safety, high efficacy, and treatment therapeutic feasibility (e.g., oral administration, blood-brain barrier [BBB] penetration). In particular, despite robust efforts using central administration of this type of drug, it is not been possible to overcome the huge barrier associated with drug penetration through the $\mathrm{BBB}$, which severely limits clinical applications.

In the present study, we evaluated 11 TCMC compounds (TCMCs), a series of synthetic MC4R antagonists, using 3 rat models: (a) LPS-induced acute anorexia, (b) cancer cachexia induced by methylcholanthrene (MCA) sarcoma, and (c) CKD-associated cachexia induced by $5 / 6$ subtotal nephrectomy. Particularly, with a number of pilot studies, we selected TCMCB07 from 11 TCMCs and tested its efficacy via a series of comprehensive approaches. Our results demonstrate that both central and peripheral treatment of TCMCB07 via 4 administration routes ([a] i.c.v. injection, [b] i.p. injection, [c] s.c. injection, and [d] oral [intragastric] gavage) increase food intake, attenuate body weight loss, and preserve fat mass and lean mass. In addition, peripheral TCMCB07 treatment diminishes hypothalamic inflammation in cancer cachexia. This preclinical trial suggests that TCMCBO7 is a promising drug candidate with a high efficacy for ameliorating cachexia, indicating this is a potential target for treatment of patients with cancer, CKD, and infectious disease.

\section{Results}

Compound and dose selection. In order to select the most effective drug candidate and determine a safe and minimal effective dose for a subsequent comprehensive evaluation, we initially performed dose-response experiments with a series of $11 \mathrm{com}$ pounds (TCMCB01-10 and a deamidated version of 1 compound, TCMCB7A) using an acute LPS model (Supplemental Table 1; supplemental material available online with this article; https:/doi. org/10.1172/JCI138392DS1). LPS doses (100-250 $\mu \mathrm{g} / \mathrm{kg} / \mathrm{d})$ were determined based on the results of a dose-response study (Supplemental Figure 1) and others' reports (43). Due to prior research showing that synthetic MC4R antagonists are most effective when given directly into the CNS, central administration via i.c.v. injection was chosen for test initiation of all 11 TCMCs. The dose of 2 $\mathrm{g} / \mathrm{rat} / \mathrm{d}(1.5 \mathrm{nmol} / \mathrm{rat} / \mathrm{d})$ was selected for i.c.v. injection. Consequently, $1.1-3 \mathrm{mg} / \mathrm{kg} / \mathrm{d}$ was selected for i.p. or s.c. injection and 6-12 mg/kg/d for oral gavage. Results from initial tests validated that TCMCB07 has the most robust positive effects on both stimulation of appetite and attenuation of body weight loss in LPS-treated rats. Furthermore, TCMCBO7 has the best solubility among 11 TCMCs. Therefore, TCMCB07 was selected for further evaluation. Specificity of TCMCBO7 to the MCR3R and MC4R was characterized by EuroscreenFast. For the MC3R, the $\mathrm{IC}_{50}$ was 11.1 nmol and the Hill coefficient was 0.93. For the MC4R, the $\mathrm{IC}_{50}$ was $31.5 \mathrm{nmol}$ and the Hill coefficient was 1.22.

Central and peripheral administration of TCMCBO7 attenuates anorexia and body weight loss induced by LPS. We first examined the effects of central administration of TCMCBO7 on food intake and body weight gain in rats with LPS (Figure 1A). LPS-treated rats receiving i.c.v. injection of TCMCBO7 at $2 \mu \mathrm{g} / \mathrm{rat} / \mathrm{d}(1.5 \mathrm{nmol} /$ rat/d) significantly increased 24-hour food intake compared with LPS-treated rats receiving saline injection (Figure 1B). Consequently, 24-hour body weight loss was attenuated in the LPS/ TCMCB07-i.c.v. group compared with the LPS/saline-i.c.v. group (Figure 1C). Similar results were observed from a subset control experiment with LPS-treated rats receiving i.c.v. injection of the MCR4 antagonist SHU9119 (1.5 nmol/rat/d) or TCMCBO2 (1.5 nmol/rat/d, Supplemental Figure 2, A and B). To evaluate effects of peripheral administration of TCMCBO7 in rats with LPS-induced acute illness, we performed i.p. injection and intragastric gavage with TCMCBO7 in LPS-treated rats through separate experiments and then measured food intake at multiple time points within 24 hours or 48 hours (Figure 1A). There was no difference in nocturnal food intake at the first 2 time points ( 2 hours and 4 hours) after i.p. injection between the LPS/TCMCB07-i.p. (3 $\mathrm{mg} / \mathrm{kg} / \mathrm{d}$ ) and LPS/saline-i.p. groups (Figure 1D), but cumulative food intake at the 16-hour and 24-hour time points was significantly different between the LPS/saline-i.p. and LPS/TCMCB07i.p. groups (Figure 1D). The 24-hour body weight loss in the LPS/ TCMCB07-i.p. group was attenuated compared with that of the saline-treated group (Figure $1 \mathrm{E}$ ). To compare with a previously reported derivative-of SHU9119 (PG932) (42), we performed a subset i.p. experiment with PG932 and TCMCBO3 and found that both PG932 and TCMCB03 had no positive effects on LPS-treated rats (Supplemental Figure 2, C and D). Based on the positive effects of TCMCBO7 through i.c.v. and i.p. administration, we next investigated whether oral administration of TCMCBO7 had similar effects. Intragastric gavage twice daily with water or TCMCBO7 $(10 \mathrm{mg} / \mathrm{kg} / \mathrm{d})$ was performed in LPS-treated rats (Figure 1A). Food intake after treatment at 24 hours (days 0-1), but not 4 hours, was significantly increased in the LPS/TCMCB07-gavage group compared with the LPS/water-gavage group (Figure 1, F and $\mathrm{G}$ ), and there was no difference at either baseline (previous day of treatment) on days 1-2 (the second day after treatment, Figure $1 G)$. There was a difference in body weight gain between 2 groups at the 24-hour time point after treatment but not at baseline or on days $1-2$ after treatment (Figure $1 \mathrm{H}$ ).

Central administration of TCMCBO7 attenuates cancer cachexia. Previous studies demonstrated that cancer cachexia can be attenuated by genetic deletion of MC4R or pharmacologic blockade of MC4R signaling using peptide antagonists, such as AgRP and SHU9119, administered i.c.v. $(18,44)$. Consistent with this, our data showed that cachexia associated with MCA sarcoma was substantially attenuated by i.c.v. administration of AgRP. Our previous 

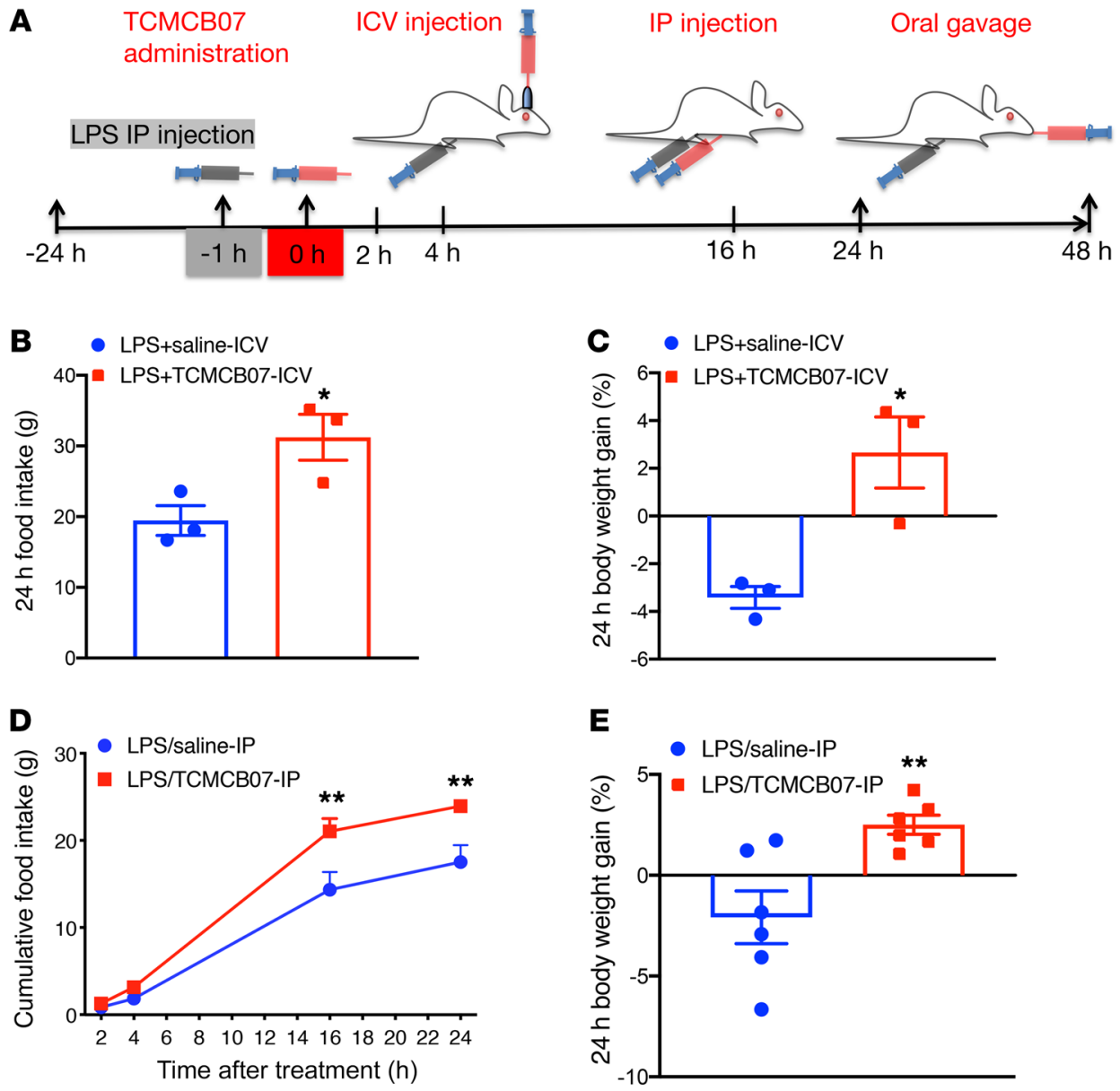

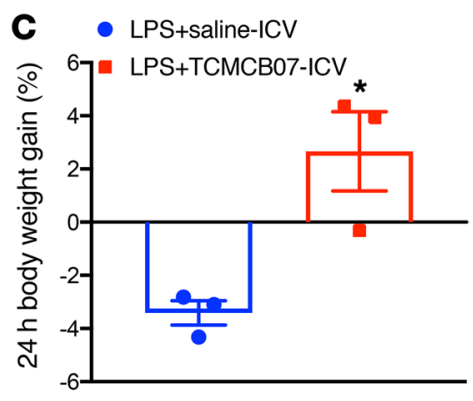

E

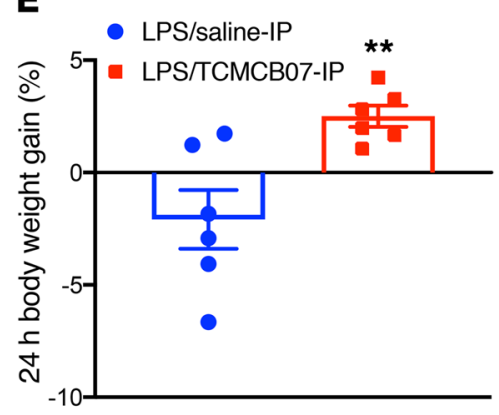

Figure 1. Central and peripheral administration of TСMСBO7 attenuates anorexia and body weight loss in rats treated with LPS. (A) Schematic of LPS injection, TCMCB07 administration, and time points of measurements. (B) Twenty-four-hour food intake and (C) 24-hour body weight gain (\%, net gain normalized to baseline) in LPS-treated rats after i.c.v. injection of saline $(n=$ 3) or TCMCBO7 (1.5 nmol/rat/d, $n=3)$. (D) Cumulative food intake at 2 hours, 4 hours, 16 hours, and 24 hours, and (E) 24-hour body weight gain (\%) in LPS-treated rats after i.p. injection of saline $(n=6)$ or ТСМСВ07 ( $3 \mathrm{mg} / \mathrm{kg} / \mathrm{d}$, $n=6$ ). (F) Cumulative food intake at 4 hours and 24 hours after oral gavage, (C) 24-hour cumulative food intake before and after oral gavage, and $(\mathbf{H})$ 24-hour body weight gain (\%) after oral gavage of water $(n=9)$ or TCMCВ07 (10 $\mathrm{mg} / \mathrm{kg} / \mathrm{d}, n=10$ ) in LPS-treated rats. All data in B, C, and E-H are expressed with each dot representing 1 sample, and data in $\mathbf{D}$ are expressed as mean \pm SEM for each group. ${ }^{*} P<0.05 ;{ }^{* *} P<$ $0.01 ;{ }^{* *} P<0.001$, unpaired Student's $t$ test (B, C, and E); 2-way ANOVA (D, F, $\mathbf{G}$, and $\mathbf{H})$.

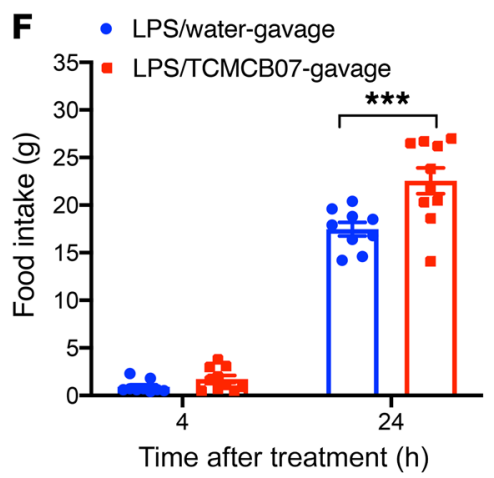

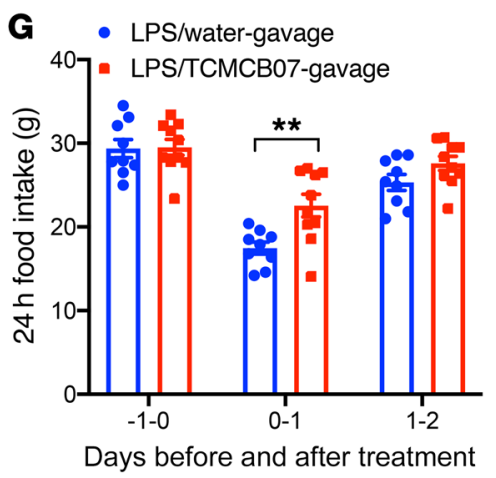

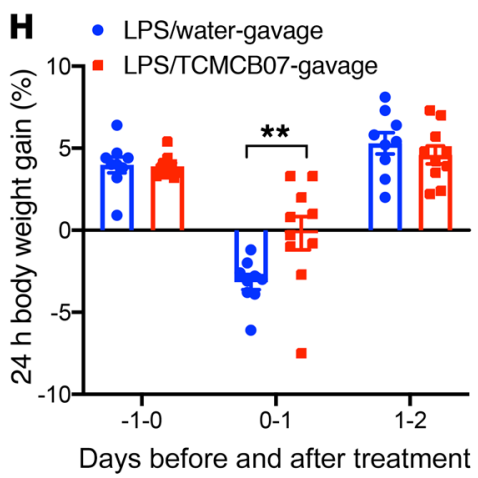

studies and others' reports validated that MCA sarcoma produces reliable, reproducible, and consistent cancer cachexia recapitulating key characteristics of the clinical condition $(22,34,45)$. Using this model, we tested the efficacy of central administration of TCMCBO7 in reversing cancer cachexia (Figure 2A). Six days after tumor implantation, tumors became palpable and tumor-bearing rats started decreasing food intake due to rapid tumor growth and cachexia development and then experienced sustained decline in food intake for the rest of the experimental period (Figure 2B). Starting at day 8 , all tumor-bearing rats received i.c.v. injection with either saline or TCMCBO7 $(1.5 \mathrm{nmol} / \mathrm{rat} / \mathrm{d})$ at $4 \mathrm{pm}$ once daily for a total of 4 consecutive days. As a result of i.c.v. injection of TСМСВ07, food intake was increased in the tumor/TCMCB07 group following
2 doses of TCMCBO7 treatment and the increase was sustained for the next 2 days while animals received TCMCBO7 injections (Figure 2B). In contrast, a sustained decline in food intake was exhibited in the tumor/saline group (Figure 2B). In comparison with TCMCBO7 treatment, i.c.v. administration of AgRP showed similar effects in a parallel study (Figure 2C). Consequently, there was a significant difference in cumulative food intake between the 2 treatment groups (saline versus TCMCBO7 or saline versus AgRP, Figure 2, D and E). Body weights (before and after tumor removal) in the tumor/ TCMCBO7 group and the tumor/AgRP group were greater than those in the tumor/saline group (Supplemental Figure 3 and Figure 2, F and G), but no difference was found in tumor mass (Figure 2, $\mathrm{H}$ and $\mathrm{I}$. Tumor-bearing rats treated with saline lost substantial fat 
A

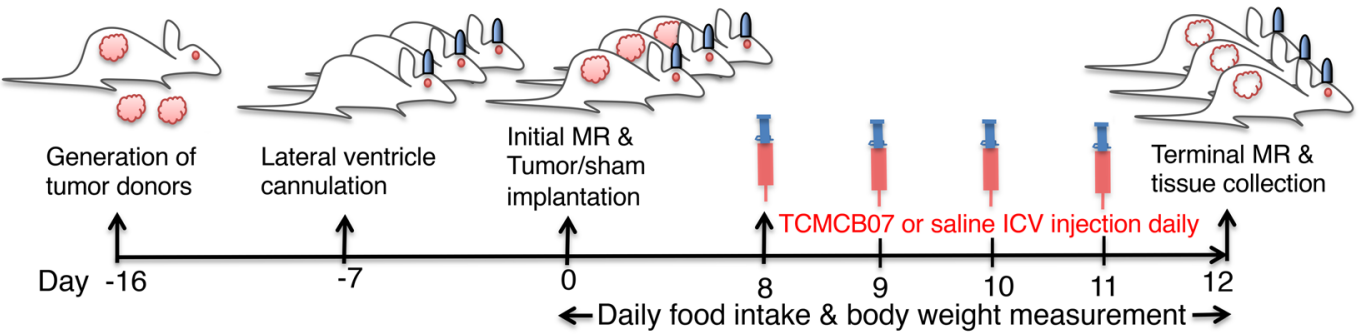

TCMCB07 treatment

B

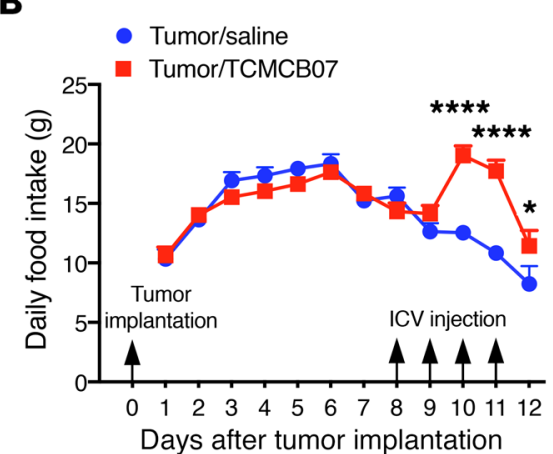

D

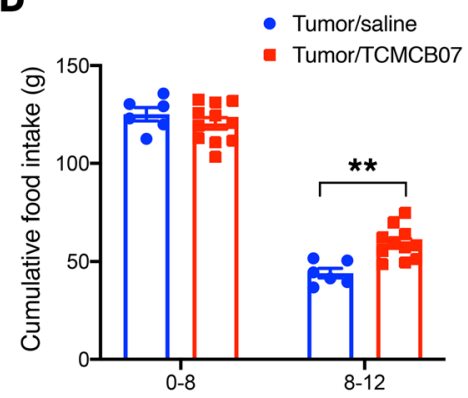

Days before and after treatment
C

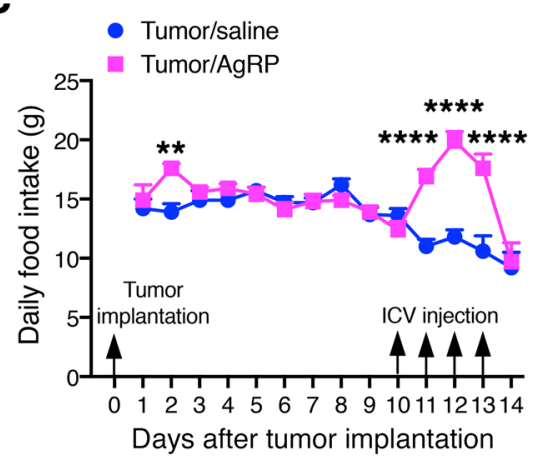

E

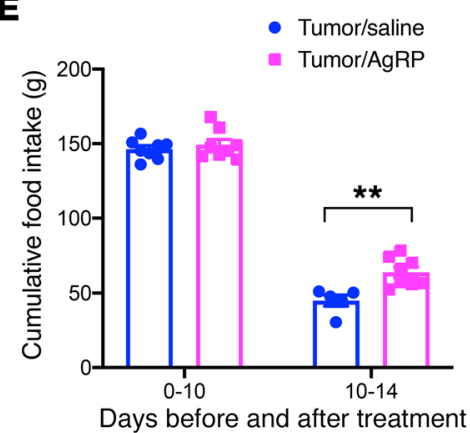

Figure 2. Central administration of TCMCB07 that is similar to AgRP treatment attenuates anorexia and body weight loss in rats with cancer cachexia.

(A) Schematic of experimental design. Tumor donors were generated approximately 16 days before tumor implantation. Brain unilateral ventricle cannulation was performed, and animals were allowed to recover for a minimum of 7 days before tumor implantation. Fresh tumor tissue from donors was implanted into F344 rats. Daily food intake and body weights were measured after tumor implantation. Injections of saline or TCMCBO7 i.c.v. were performed between days 8 and 11 after tumor implantation. Body composition (initial and terminal) was measured by MRI. (B) Daily food intake in tumor-bearing rats receiving i.c.v. injection once daily with saline $(n=6)$ or TCMCBO7 $(1.5 \mathrm{nmol} /$ rat/d, $n=11$ ). (C) In a separate experiment, tumor-bearing rats received i.c.v. injection once daily with saline ( $n$ $=8$ ) or AgRP ( $1 \mathrm{nmol} / \mathrm{rat} / \mathrm{d}, n=8$ ) between days 10 and 13 after tumor implantation. ( $\mathbf{D}$ and $\mathbf{E}$ ) Cumulative food intake before and after compound treatment. (F and $\mathbf{G}$ ) Body weight gain (\%, tumor-free net gain normalized to baseline) before and after treatment. ( $\mathbf{H}$ and $\mathbf{I})$ Tumors were dissected and weighed after animals were euthanized on day 12 or day 14 . ( $\mathbf{~ a n d} \mathbf{K}$ ) Fat mass and ( $\mathbf{L}$ and M) lean mass were determined by MRI on day 0 and day 12 or day 14 , and gain was calculated (\%, net gain normalized to baseline). All data in $\mathbf{B}$ and $\mathbf{C}$ are expressed as mean \pm SEM for each group. All data in $\mathbf{D}-\mathbf{M}$ are expressed with each dot representing 1 sample. ${ }^{*} P<$ 0.05 ; ${ }^{* *} P<0.01$; ${ }^{* * *} P<0.001$; ${ }^{* * *} P<0.0001$, 2-way ANOVA (B-G); unpaired Student's $t$ test (H-M).
TCMCB07 treatment

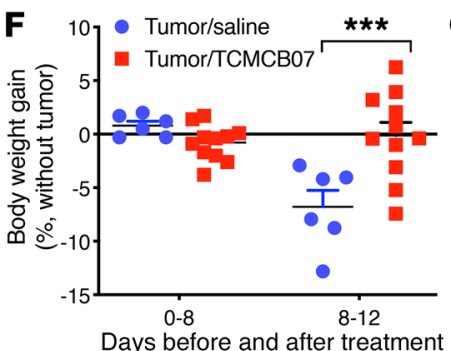

H

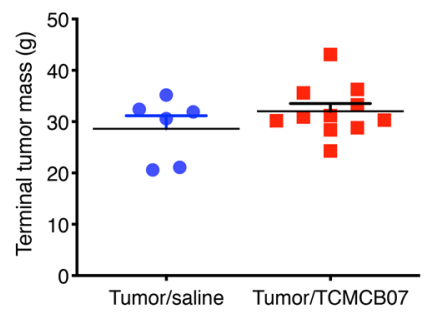

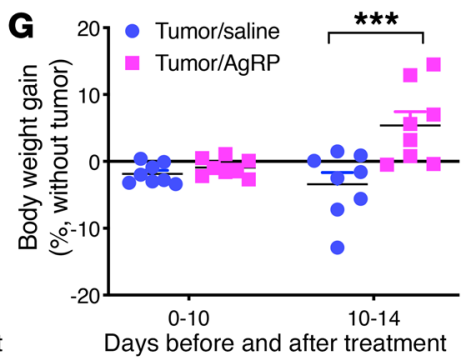

1

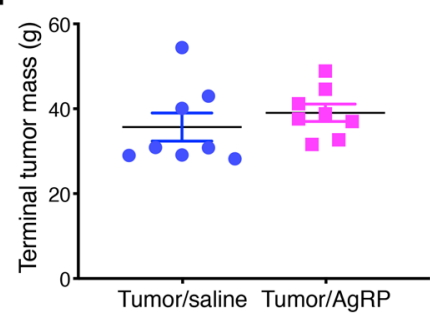

TCMCB07 treatment

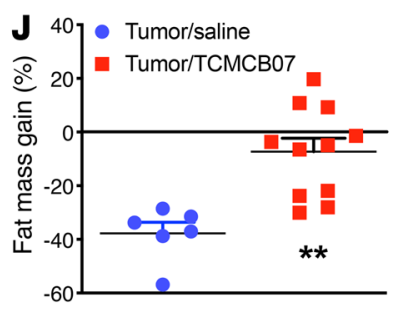

$\mathbf{L}$

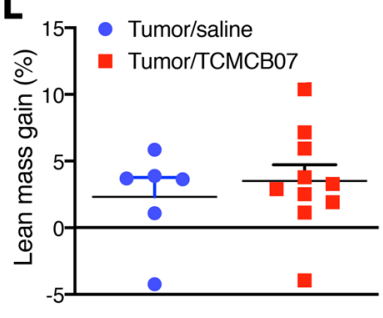

AgRP treatment
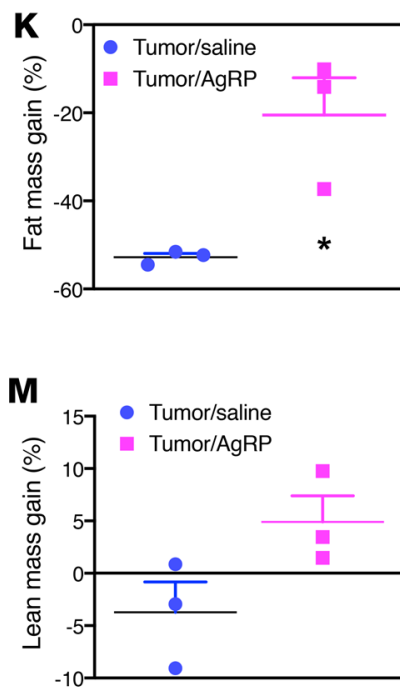
$\mathbf{A}$
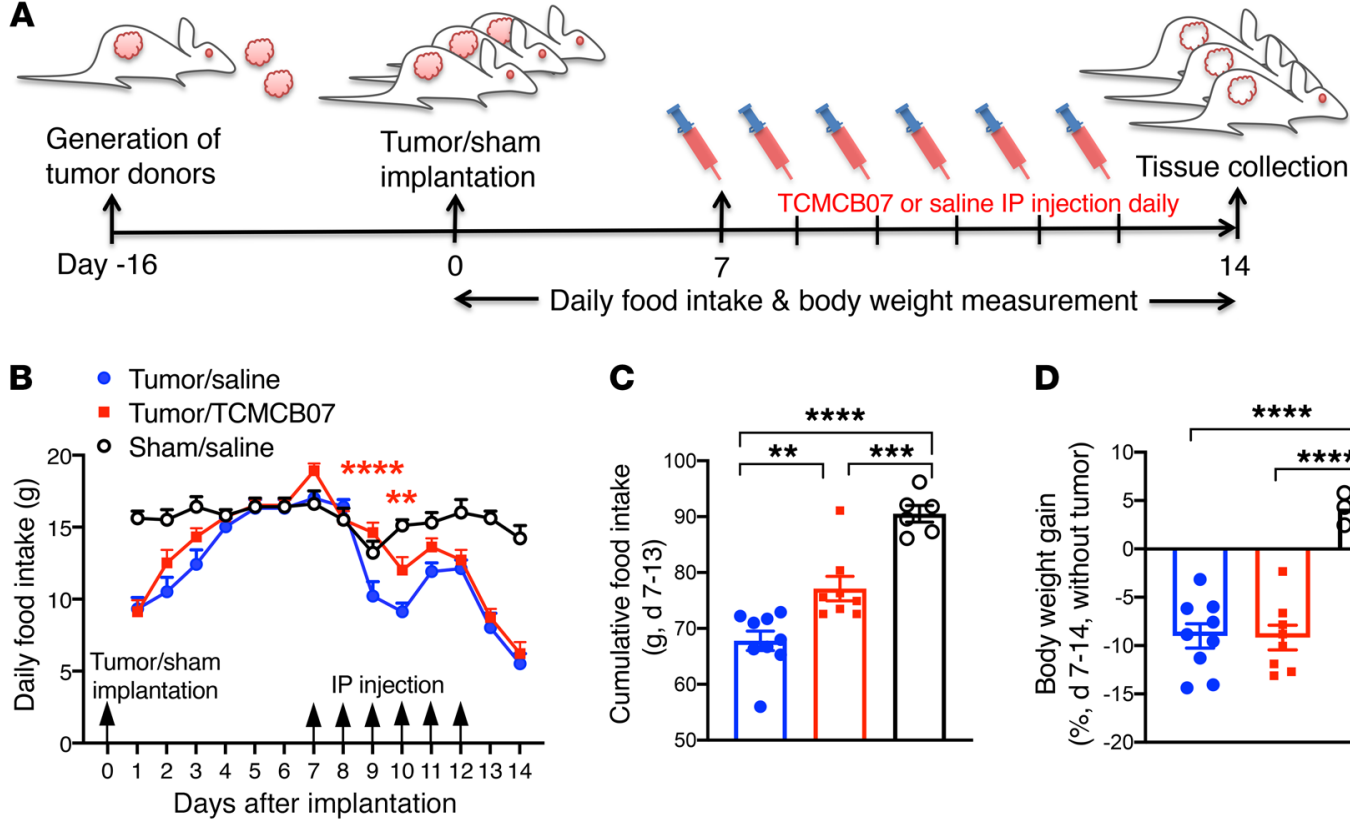

C

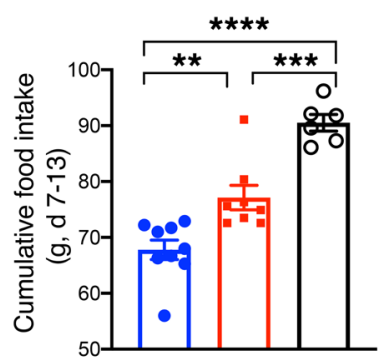

D

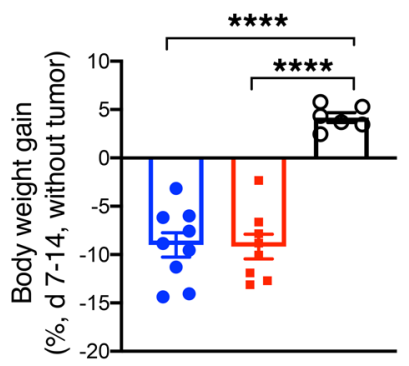

E

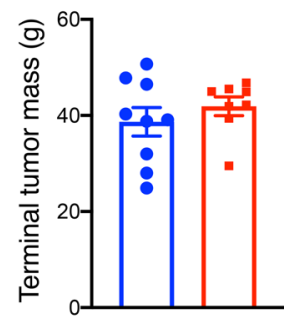

Figure 3. Peritoneal administration of ТСМСВ07 diminishes anorexia in rats with cancer cachexia. (A) Schematic of experimental design. (B) Daily food intake in tumor/saline group $(n=9)$, tumor/TCMCB07 group $(n=8)$, and sham/saline group $(n=6)$ after tumor implantation or sham operation. Rats received i.p. injection once daily with saline or TСMСBO7 ( $3 \mathrm{mg} / \mathrm{kg} / \mathrm{d}$ ) between days 7 and 12 after implantation. (C) Cumulative food intake after saline or TCMCB07 treatment. (D) Body weight gain (\%, tumor-free net gain normalized to baseline of day 7 ) after saline or TCMCB07 treatment. (E) Tumors were dissected and weighed after animals were euthanized on day 14. All data in B are expressed as mean \pm SEM for each group, and all data in C-E are expressed with each dot representing 1 sample. Two-way ANOVA in B and sham/saline group was excluded from 2-way ANOVA analysis in order to clearly show a treatment comparison between 2 tumor groups (tumor/TCMCB07 versus tumor/saline). ${ }^{* *} P<0.01 ;{ }^{* *} P<0.001$; ${ }^{* * * *} P<0.0001,1$-way ANOVA (C and D); unpaired Student's $t$ test (E). Red asterisks in B indicate tumor/TCMCB07 group versus tumor/saline group.

mass compared with the initial baseline, whereas tumor-bearing rats treated with TCMCBO7 or AgRP preserved a significant amount of fat mass (Figure 2, J and K). There was a trend toward greater preservation of lean mass in the tumor/TCMCB07 or tumor/AgRP group, but it did not reach statistical significance (Figure 2, L and M).

Administration of TCMCBO7 i.p. ameliorates cancer-associated anorexia. We next evaluated the consequences of peripheral administration of TCMCBO7 on cancer cachexia through i.p. injection. Starting at day 7, tumor-bearing rats received i.p. injection once daily with either saline or TCMCBO7 $(3 \mathrm{mg} / \mathrm{kg} / \mathrm{d})$ for a total of 6 consecutive days (Figure $3 \mathrm{~A}$ ). After 2 doses of treatment, tumor-bearing rats receiving TCMCB07 exhibited greater food intake compared with the tumor/saline group (Figure 3B). Accordingly, 6-day cumulative food intake after treatment in the tumor/TCMCB07 group was significantly greater than that in the tumor/saline group (Figure $3 \mathrm{C}$ ). These data suggest that i.p. administration of TCMCBO7 effectively stimulates appetite during cancer cachexia. However, we did not find a difference in body weights between the 2 treatment groups (Supplemental Figure 4 and Figure 3D). Tumor weights between the 2 treatment groups were identical (Figure 3E). There was a significant change in food intake and body weight gain after implantation in tumor-bearing rats versus sham-operation rats (Figure 3, B-D), representing key features of this cachexia model.

Administration of TCMCBO7 s.c. ameliorates cancer cachexia. Because neither central nor peritoneal drug delivery is convenient or feasible for clinical application, we further sought an alternative peripheral route to deliver TCMCB07. Administration s.c. is a feasible clinical option and might permit a lower dose of $\mathrm{TCMCBO} 7(1.5 \mathrm{mg} / \mathrm{kg} / \mathrm{d})$ treatment. We split 1 dose into 2 injections that were administered in early morning and later evening to maintain a sustained effective drug concentration. Similarly to what was observed with i.p. injection, after 2 day of s.c. treatment, tumor-bearing rats receiving ТСМCBO7 at either a high dose $(3 \mathrm{mg} / \mathrm{kg} / \mathrm{d})$ or a low dose $(1.5 \mathrm{mg} / \mathrm{kg} / \mathrm{d})$ significantly increased food intake compared with saline-treated tumor rats, and the increase remained for the rest of the experimental period while animals continually received TCMCB07 injection (Figure 4A). Six-day cumulative food intake in tumor rats treated with TCMCB07 (low dose or high dose) was remarkably greater than that in tumor rats treated with saline. There were no differences between groups before treatment (Figure $4 \mathrm{~B})$. Furthermore, a trend toward dose-dependent increase in food intake was observed (Figure 4, A and B). There was no difference in 6-day cumulative food intake after treatment between tumor/TCMCB07H and sham/saline groups (Figure $4 \mathrm{~B})$. Accompanying this positive effect on food intake, tumorbearing rats receiving TCMCB07 s.c. injections, particularly at a high dose, maintained body weight relative to saline-treated tumor rats (Supplemental Figure 5 and Figure 4C). There was significant body weight loss among all tumor-bearing animals compared with sham-treated animals, but the degree was different between TCMCBO7 treatment groups and the saline group (Figure 4C). No differences were found in tumor mass among the 3 tumor groups (Figure 4D). At the end of the experiment, 
A
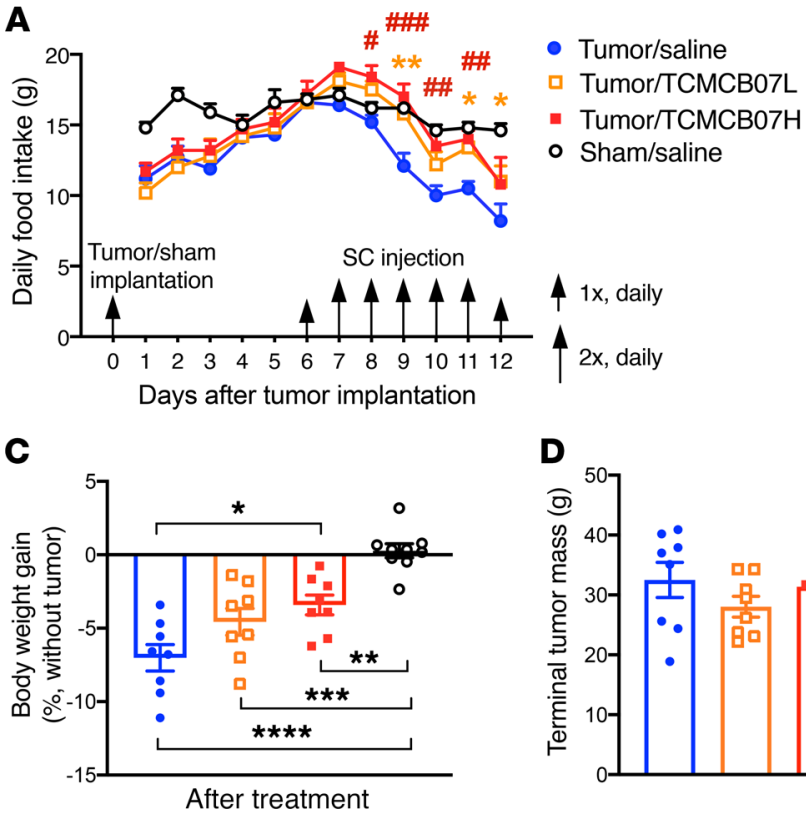

B

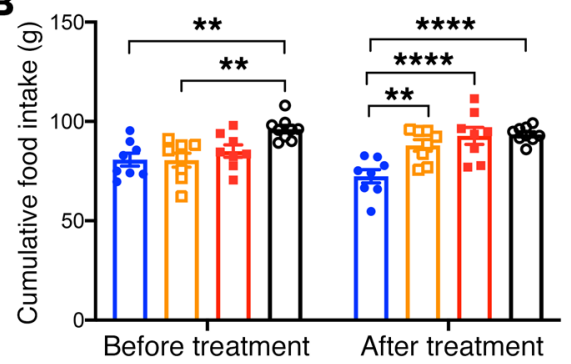

$\mathbf{E}$

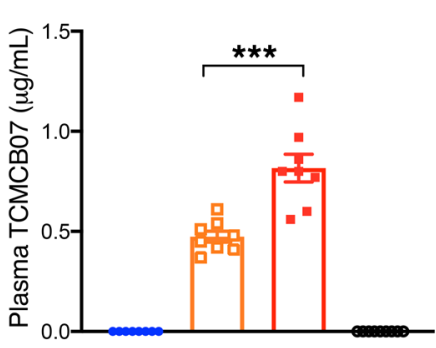

Figure 4. Administration of TСMCB07 s.c. ameliorates anorexia and body weight loss in rats with cancer cachexia. (A) Daily food intake in tumor/saline group $(n=8)$ versus tumor/TCMCB07L (low dose) group $(n=8)$, tumor/TCMCBO7H (high dose) group $(n=8)$, and sham/saline group ( $n=9)$ after tumor implantation or sham operation. Rats received s.c. injection once (1x) or twice (2x) daily with saline or TCMCB07L (1.5 mg/kg/d) or TCMCB07H (3 mg/kg/d) between day 6 and day 12 after implantation. (B) Cumulative food intake before and after treatment. (C) Body weight gain after treatment (\%, tumor-free net gain normalized to baseline of day 6). (D) Tumors were dissected and weighed after animals were euthanized on day 12. (E) Terminal plasma TCMCB07 concentrations were measured by reverse-phase HPLC. All data in $\mathbf{A}$ are expressed as mean \pm SEM for each group, and all data in B-E are expressed with each dot representing 1 sample. Two-way ANOVA in $\mathbf{A}$ and for sham/saline group was excluded for 2-way ANOVA analysis in order to clearly show a treatment comparison among 3 tumor groups (tumor/saline, tumor/TCMCB07L, tumor/TCMCB07H) (A). ${ }^{*} P<0.05 ;{ }^{* *} P<0.01 ;{ }^{* *} P<0.001 ;{ }^{* * * *} P<0.0001$; ${ }^{\#} P<0.05 ;{ }^{\#} P<0.01 ; \# \# \#<0.001$, 1-way ANOVA (B-D); unpaired Student's $t$ test (E). Yellow asterisks in A show tumor/TCMCB07L group versus tumor/ saline group, and red pound signs in A show tumor/TCMCBO7H group versus tumor/saline group.

blood was collected from all animals via cardiopuncture and plasma was assayed for ТСМСВ07 concentration by investigators who were blinded to group information. All animals receiving ТСМСВ07 but not saline had detectable plasma ТСМСВ07 levels that were tightly correlated with the administered doses (Table 1). Plasma TCMCBO7 concentration in the tumor/ TCMCB07H group was nearly 2 -fold higher than that in the tumor/TCMCB07L group, which replicated the dose ratio (high dose versus low dose: 3 versus $1.5 \mathrm{mg} / \mathrm{kg} / \mathrm{d}$ ) (Figure $4 \mathrm{E}$ ).

Administration of TCMCBO7 s.c. reverses cachexia associated with CKD. To validate whether ТСМСB07 has a universal efficacy in reversing cachexia associated with various conditions, we used a common nonmalignancy cachexia model, CKD-related renal failure induced by surgical 5/6 subtotal nephrectomy (Figure $5 \mathrm{~A}$ ). After a week (days 0-7) of recovery from stage I nephrectomy (unilateral partial nephrectomy [Neph-I]) or sham operation (sham), all surgical rats gained a similar amount of body weight (Figure 5B). After a week (days 7-14) of recovery from stage II nephrectomy (contralateral total nephrectomy, Neph-II) or sham operation, all nephrectomy (neph) rats lost a notable amount of body weight compared with the sham group (Figure 5B), but there was no difference between the 2 neph groups (Figure 5C). With s.c. administration of TCMCB07 twice daily $(3 \mathrm{mg} / \mathrm{kg} / \mathrm{d})$, neph rats persistently gained body weight over the 14-day treatment period and finally caught up to the sham/saline group (Figure 5B). However, neph rats receiving saline treatment gained weight more slowly (Figure 5B). Total body weight gain after 14 days of treatment was significantly less in the neph/saline group than in the neph/TCMCB07 group (Figure 5C). As expected, daily food intake in neph rats receiving ТСМСВ07 was higher than that in the neph/saline group and was similar to that in the sham/saline group (Figure 5D). Cumulative food intake was significantly different between neph/saline and neph/ТСМСB07 groups for the entire 14-day treatment (Figure 5E). Remarkably, 14-day s.c. administration of ТСМСB07 reversed both fat mass and lean mass loss in neph rats (Figure 5, $\mathrm{F}$ and $\mathrm{G}$ ).

To confirm renal failure and drug distribution, plasma was assayed at the end of the study for concentration of blood urea nitrogen (BUN), creatinine (Cr), and TCMCB07. Both BUN and $\mathrm{Cr}$ were increased in all neph rats relative to sham rats, consistent with chronic renal failure, and there was no difference between the neph/saline and neph/TCMCB07 groups (Figure 6, A and B). TCMCBO7 concentration in plasma was detectable among all rats receiving ТСMCB07 s.c. injection (Table 1), and undetectable in all rats receiving saline (Figure $6 \mathrm{C}$ ). Correlation analysis suggested that 14-day food intake was not associated with plasma BUN levels (Figure 6D), but it negatively correlated with plasma Cr levels (Figure $6 \mathrm{E})$. Both 14-day food intake and body weight gain were positively correlated with plasma TCMCB07 levels (Figure 6, F and G).

Administration of TCMCBO7 s.c. diminishes hypothalamic inflammation in cancer cachexia. Because excessive inflammation is a key driver for cachexia, we determined whether s.c. administration of 


\section{Table 1. ТСMCB07 doses and plasma concentrations in Cachexia-associated disease}

\section{Cachexia-associated disease}

Cancer

Cancer

CKD

TCMCB07 doses
(mg/kg/d, s.c. injection
1.5
3.0
3.0

3.0

\author{
TCMCB07 plasma \\ concentrations $(\mu \mathrm{g} / \mathrm{mL})$ \\ $0.47 \pm 0.03$ \\ $0.82 \pm 0.07$ \\ $1.37 \pm 0.12$
}

At the end of experiments, plasma was collected after rats were euthanized. Plasma TCMCB07 concentrations were assayed using reverse-phase HPLC. Rats with cancer- and CKD-associated cachexia were treated with TCMCB07 at low dose $(1.5 \mathrm{mg} / \mathrm{kg} / \mathrm{d})$ or high dose $(3.0 \mathrm{mg} / \mathrm{kg} / \mathrm{d})$ via s.c. injection. Rats with cancer cachexia received 12 injections (both low-dose and high-dose groups, $n=8)$ in the last 7 consecutive days. Rats with CKD-associated cachexia $(n=11)$ received 28 injections (high dose) in the last 14 consecutive days. Data for the plasma concentrations are expressed as mean \pm SEM for each group.

TCMCB07 attenuates hypothalamic inflammation associated with cachectic conditions. As we previously observed in this model as well as other cancer cachexia models $(28,34)$, there was a significant upregulation in inflammatory gene expression of Il1b, Illr1, and Il6, but not Tnf, in tumor-bearing rats relative to sham-treated rats (Figure 7A). Indeed, s.c. administration of TCMCB07 suppressed the expression of Il1b, Illr1, and Il6 in tumor-bearing rats compared with those that received saline treatment (Figure 7A). Selp, a gene encoding P selectin, has been linked to development of cancer cachexia (46). We observed highly upregulated Selp in tumor-bearing rats compared with sham-treated rats, which was also found in other cancer cachexia models and cancer patients $(28,46)$. Interestingly, TCMCBO7 treatment dramatically suppressed Selp gene expression in tumor-bearing rats compared with rats receiving saline treatment (Figure 7A). These data suggest that s.c. administration of TCMCBO7 suppresses hypothalamic inflammation, and this may contribute to its beneficial effects during cachexia. In the CKD model, there was a trend toward increases in gene expression of Il1b, Illr1, Il6, Tnf, and Selp in neph rats compared with sham-treated rats, but none reached statistical significance (Figure 7B). Furthermore, there was no difference in Pomc gene expression between tumor or neph and sham-treated rats, although there was a trend toward decrease of the expression in tumor/saline rats. Compared with what occurred in sham-treated rats, Agrp gene expression was upregulated in cachexia rats, particularly in those with cancer (Figure 7, A and B). Remarkably, TCMCB07 treatment suppressed Agrp upregulation in both tumor and $\mathrm{CKD}$ animals, which resulted in a transcriptional level similar to that found in sham-treated animals (Figure 7, A and B).

\section{Discussion}

Over the last two decades, several research groups contributed to the development of orexigenic agents to treat cachexia, including MC4R antagonists and ghrelin analogs. Our lab evaluated a number of drug candidates and demonstrated that some of them had promising effects in amelioration of cachexia associated with cancer, CKD, and heart failure and LPS-induced acute anorexia, etc. $(18,33,34,40,41,47)$. Some of these agents have found their way into clinical trials in patients with cachexia $(32,35,36)$. In the present study, we evaluated efficacy of 11 TCMC MC4R antagonists, then specifically focused on TCMCB07 in 3 rat models of LPS and cancer- and CKD-associated cachexia. We sought to validate that peripheral administration of TCMCB07 was feasible for effectively inhibiting central melanocortin signaling. Our results demonstrate that peripheral treatment of TCMCBO7 has remarkably positive effects in stimulation of appetite, retention of body weight, and preservation of fat mass and lean mass under cachectic conditions. Furthermore, our data indicate that peripheral TCMCBO7 treatment attenuates hypothalamic inflammation associated with cancer cachexia. It is possible that this is an independent effect of this compound, as melanocortin signaling is known to affect inflammation $(48,49)$. However, this effect is generally associated with melanocortin agonists, and it is therefore possible that this effect is secondary to improved appetite and reduced catabolism secondary to TCMCB07 treatment.

LPS is a bacterial endotoxin and is extensively used to mimic acute infection and inflammation conditions commonly seen in patients. Based on the results of the LPS dose-response study (Supplemental Figure 1), we chose a moderate dose $(100-250 \mu \mathrm{g} / \mathrm{kg} / \mathrm{d})$ for i.p. injection to elicit reproducible sickness behaviors without extremely severe morbidity and mortality. Moreover, because we used a moderate LPS dose, we were able to observe possible side effects derived from the compounds. Other than the expected increases in food consumption, TCMCB07 administration did not produce notable behavioral alterations in the experimental rats except when given as a high dose $(20 \mu \mathrm{g} / \mathrm{rat} / \mathrm{d})$ with central administration (i.c.v. injection, Supplemental Table 1). Our data showed that both central and peripheral TCMCBO7 treatment, including oral administration, increased food intake and attenuated body weight loss in LPS-treated rats. We noted that the benefits of TCMCB07 in the acute LPS model were consistent, but delayed and not observed in the first hours after compound administration. In addition, the effective dose with i.p. injection or intragastric gavage was much higher than that with i.c.v. injection. We also tested TCMCBO7 at a very low dose $(0.3-0.6 \mathrm{mg} / \mathrm{kg} / \mathrm{d})$ through i.p. and oral routes, but did not find significant positive effects in LPS-treated rats (data not shown). Because we previously observed that repeated LPS injections in rodents can cause either desensitization or mortality, we were not able to test TCMCB07 in a setting of an LPS-induced chronic condition. Collectively, through a series of studies in the acute LPS model, we found that TCMCBO7 was the best drug candidate among the 11 TCMCs and established effective doses for both central and peripheral treatment.

Cancer cachexia is a wasting syndrome characterized by a significant reduction of body weight resulting predominantly from losses of adipose tissue and skeletal muscle $(4,6,50)$. Anorexia is often a major contributor to the weight loss and muscle wasting, and even with administration of drugs that target overactivation of catabolic processes and inflammation, adequate nutritional support still remains a mainstay of cachexia therapy (5). Appetite improvement and increased food intake can provide more nutritional support to reverse negative energy balance and promote anabolism, maintenance of body weight, and physical activity associated with quality of life and eventual survival (51). Furthermore, normalized nutritional intake can increase treatment tolerance to cancer therapy (51). Our data 


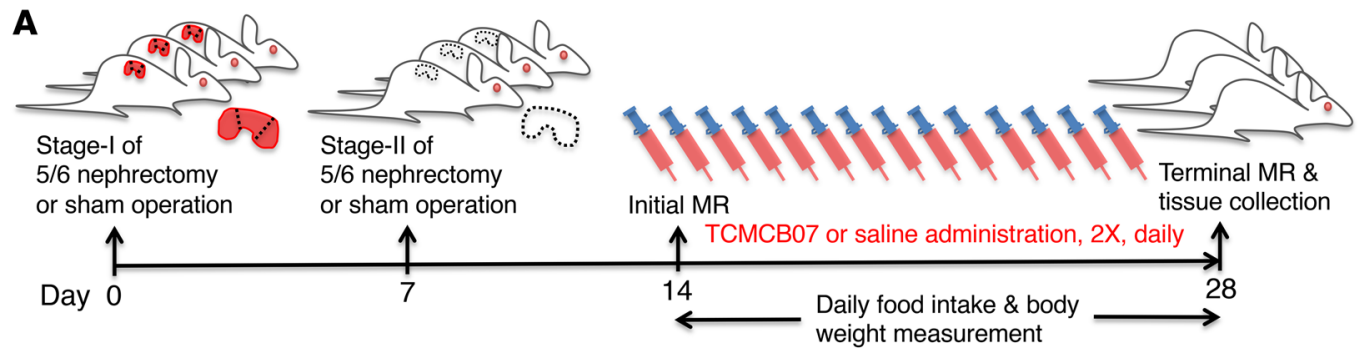

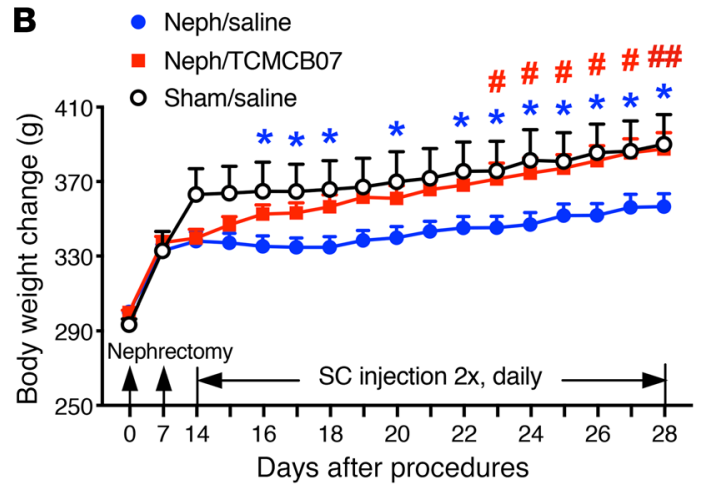

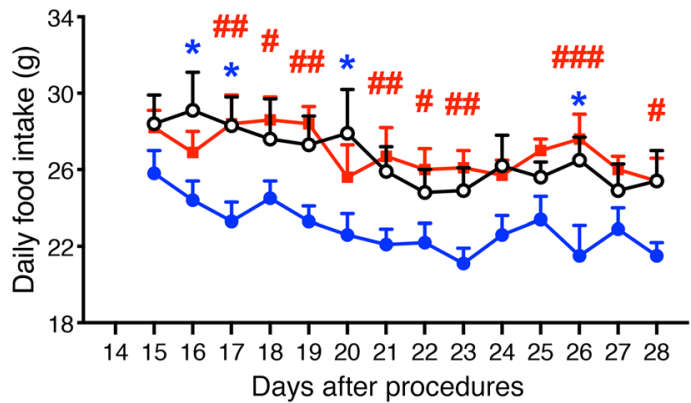

E

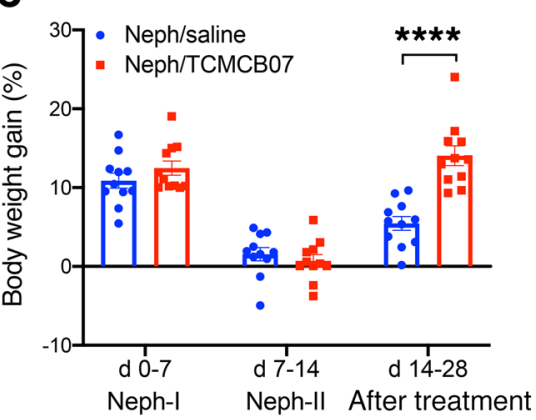

D

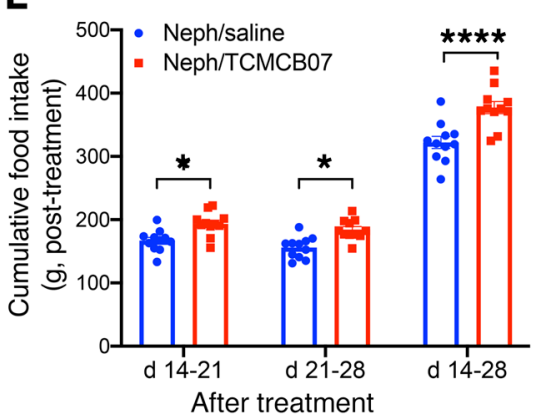

$\mathbf{F}$
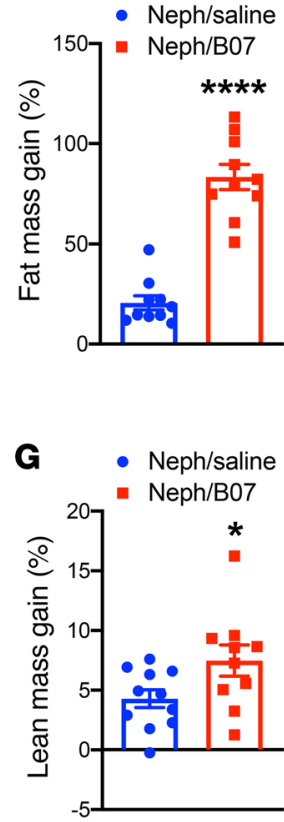

Figure 5. Administration of TСMCB07 s.c. reverses cachexia associated with CKD in rats. (A) Schematic of experimental design. Two-stage 5/6 nephrectomy or sham operation was performed in Sprague-Dawley rats. Stage I of 5/6 nephrectomy was Neph-I and stage II was Neph-II. All animals experienced Neph-I and Neph-II or sham operation on day 0 and day 7 and received s.c. injection twice (2x) daily with saline or TCMCB07 ( $3 \mathrm{mg} / \mathrm{kg} / \mathrm{d})$ between day 14 and day 28 after nephrectomy. Neph/saline group $(n=11)$, neph/TCMCB07 group $(n=11)$, and sham/saline group $(n=6)$. (B) Body weight change during entire experimental period (days 0-28, after nephrectomy). (C) Body weight gain (\%, net gain normalized to baseline) after nephrectomy and after treatment. (D) Daily food intake in neph/saline, neph/TCMCB07, and sham/saline groups after treatment. (E) Cumulative food intake after treatment. (F) Fat mass and (G) lean mass were determined by MRI before (day 14) and after (day 28) treatment, and the gain (\%) was calculated (net gain normalized to baseline). All data in $\mathbf{B}$ and $\mathbf{D}$ are expressed as mean \pm SEM for each group, and all data in $\mathbf{C}$ and $\mathbf{E}-\mathbf{G}$ are expressed with each dot representing 1 sample. ${ }^{*} P<0.05$; ${ }^{* * *} P<0.0001 ;{ }^{*} P<0.05$; \#\# $P<0.01$; ${ }^{\# \# \#} P<0.001,2$-way ANOVA (B-E); unpaired Student's $t$ test $(\mathbf{F}$ and $\mathbf{G})$. Blue asterisks in $\mathbf{B}$ and $\mathbf{D}$ indicate neph/saline group versus sham/saline group, and red pound signs in $\mathbf{B}$ and $\mathbf{D}$ indicate neph/TCMCB07 group versus neph/saline group.

demonstrate that both central and peripheral administration of TCMCB07 effectively stimulated appetite, leading to a remarkable increase in food intake during aggressive tumor growth and subsequently rapid cachexia development. It is important to note that weight gain is brought about by increased fat mass and lean mass, not water retention. As was seen with the progestational agent megestrol acetate, which increased water weight but did not increase lean mass, weight gain without lean mass gain may not improve disease outcome $(52,53)$. Our data from body composition measurement validated that there was no water retention after TСМСВ07 measurement. Furthermore, a recent retrospective study in patients with head and neck squamous cell carcinoma (HNs.c.C) demonstrated that increased BMI was associated with significantly improved survival and that decreased overall survival was predicted by skeletal muscle depletion, suggesting that decreased skeletal muscle mass or BMI can predict oncologic outcomes for patients with HNs.c.C (54).

In the cancer cachexia study with i.c.v. administration, 4 doses of TCMCBO7 (or AgRP) treatment significantly increased body weight $(P<0.001)$ and fat mass $(P<0.01)$ and produced a positive trend toward increased lean mass. A few possibilities could explain the nonsignificant lean mass gain. First, fat mass loss or gain is more rapid than lean mass loss or gain in cancer cachexia (55), and a marked increase of fat mass was observed after a short period (4 days) of treatment. It is possible that with further treatment, lean mass would have continued to accrue. Second, because of ethical considerations and the increasingly morbid nature of tumor-bearing animals, the animals had to be euthanized on days 
A

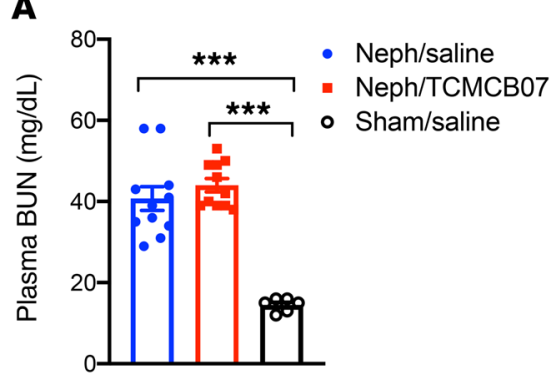

D

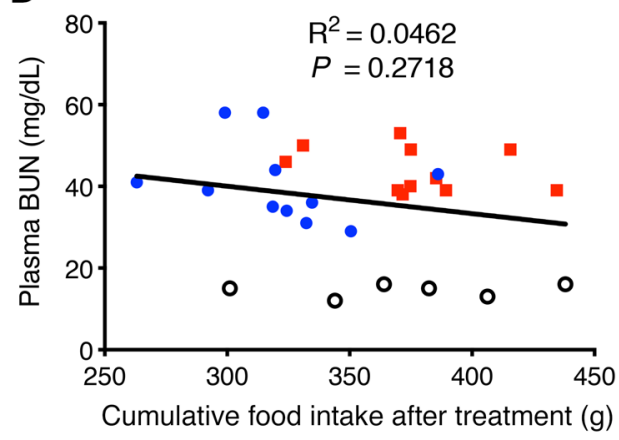

$\mathbf{F}$

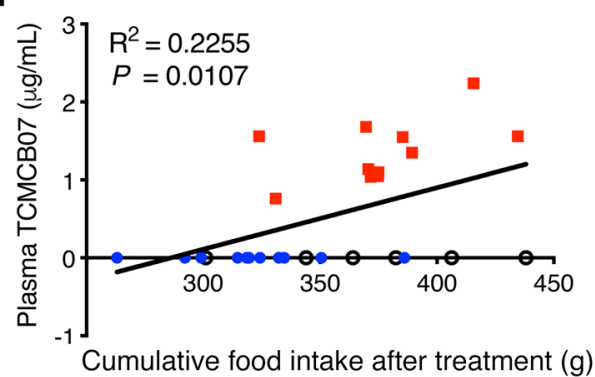

B

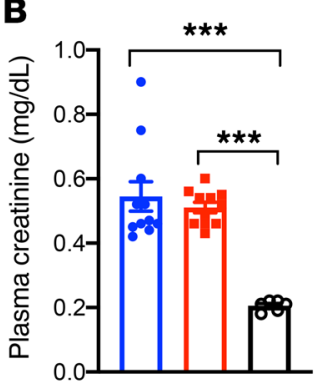

C

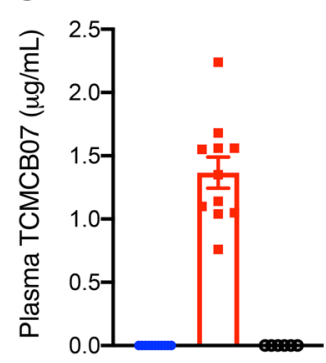

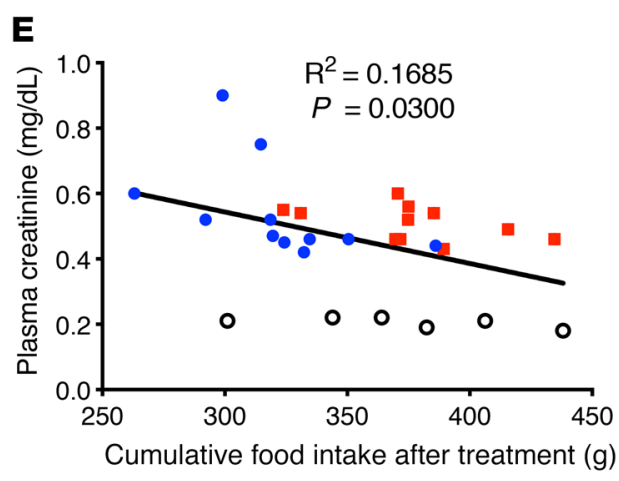

G

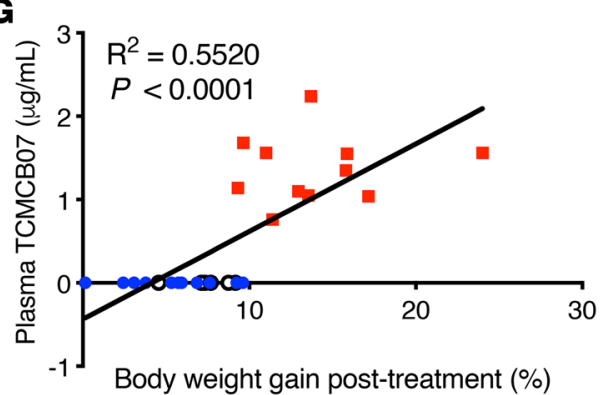

Figure 6. Plasma BUN, Cr, and TCMCBO7 concentration and the association with food intake and body weight gain in rats with CKD cachexia. Terminal plasma concentration of BUN (A), $\mathrm{Cr}$ (B), and TCMCBO7 (C) was measured after animals were euthanized. Correlation among plasma concentrations of BUN, Cr, TCMCB07, and cumulative food intake and body weight gain after treatment (D-C). All data from 3 groups (Neph/saline, $n=8$, Neph/TCMCB07, $n=8$, sham/saline, $n=6$ ) are included in the statistical analysis, with each dot representing 1 sample $(\mathbf{A}-\mathbf{G}) .{ }^{* *} P<0.001,1$-way ANOVA (A and $\left.\mathbf{B}\right)$. The correlation was analyzed using Pearson correlation coefficient, and linear regression-fitting curves are shown as black lines in D-G.

12 to 14 after tumor implantation. A less aggressive cancer type might facilitate experiments that would show long-term effects with a bigger difference in lean mass between 2 treatment groups. Another point to note is the variation caused by the complexity of the cancer cachexia model. Although in the beginning of each experiment, the same amount of fresh tumor tissue was implanted into similar locations in animals with the same sex and similar age and body size, it was difficult to control the later progression of tumor growth and subsequent cachexia. In the cancer cachexia study with i.p. administration, we extended the treatment period up to 6 doses in 6 days but did not find a difference in body weight gain between saline and TCMCB07 treatment groups. In order to maintain a sustained drug concentration in the body, in the study with s.c. administration, we split 1 dose of TCMCBO7 into 2 separate injections performed in the early mornings and evenings. It is likely that optimized dosing route, starting time point, frequency, and duration would significantly enhance drug efficacy and treatment outcomes and would facilitate lower effective doses
(3 or $1.5 \mathrm{mg} / \mathrm{kg} / \mathrm{d}$ ). It is also likely that early treatment improves the attenuation of cachexia (4). We found no association between tumor mass and TCMCB07 treatment within all cancer cachexia studies, demonstrating that increased energy intake does not lead to increased tumor growth.

Using the CKD model employed here, we previously demonstrated that treatment with ghrelin and its analogs increased food intake and lean mass and decreased circulating inflammatory cytokines in CKD-associated cachexia $(33,56)$. In the present study, renal-failure rats receiving s.c. injection of TCMCB07 twice daily consistently increased food intake and body weight, and after 14 days of treatment, body weight reached the levels found in sham-treated rats, suggesting that s.c. treatment of TCMСВ07 effectively reverses anorexia and growth failure associated with CKD. Furthermore, this relatively long-term ТСМСB07 treatment completely prevented the muscle loss normally observed with this model. These treatment outcomes are likely attributed to increased nutrient intake and improved daily physical activity. 
A

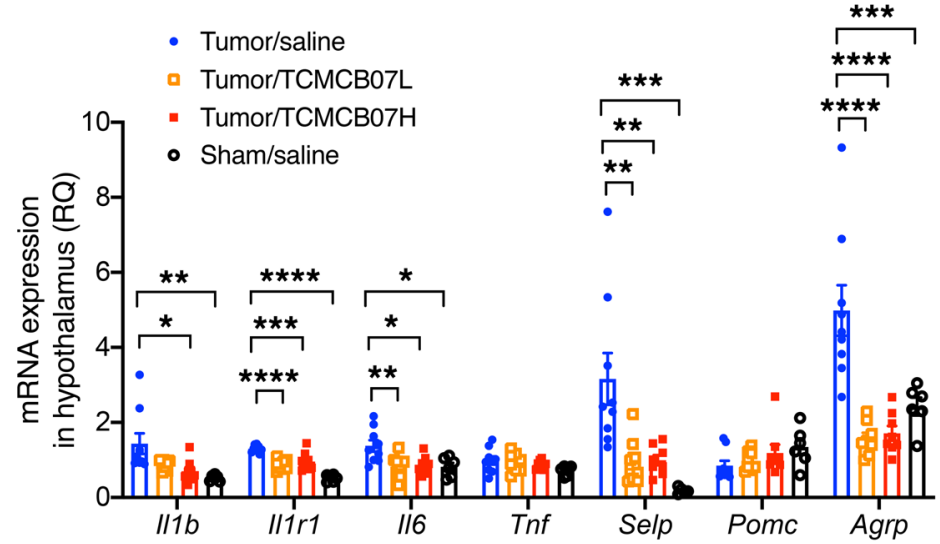

B

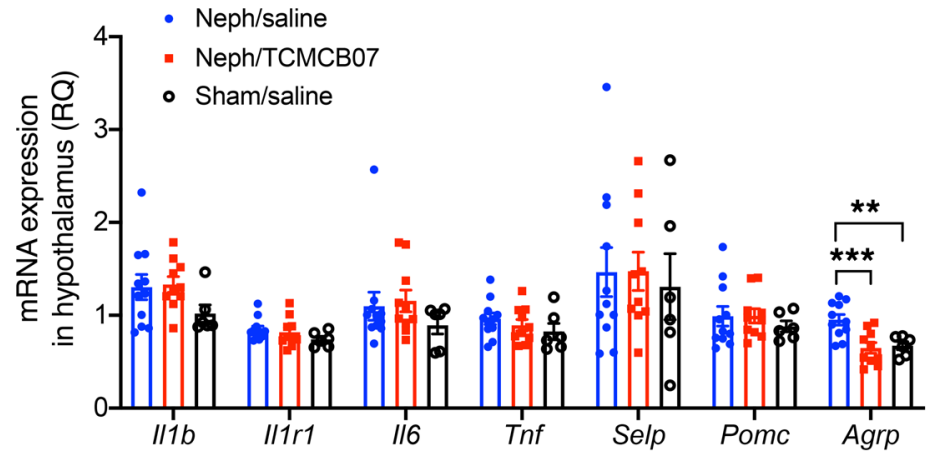

Figure 7. Treatment of ТСМСВ07 diminishes inflammatory and $\mathbf{P}$ selectin gene expression in hypothalamus in rats with cancer cachexia. The hypothalamic tissues were analyzed with qRT-PCR. Expression of inflammatory genes and anorexigenic (POMC) and orexigenic genes (AgRP) was profiled in rats with cancer cachexia (A) and CKD-associated cachexia (B) after s.c. administration of TCMCB07. Tumor/ saline group ( $n=8)$, tumor/TCMCB07L group $(n=8)$, tumor/ TCMCBO7H group $(n=8)$ and sham/saline group $(n=6)$. Neph/saline group ( $n=11)$, neph/TCMCB07 group $(n=11)$, and sham/saline group $(n=6)$. All data in $\mathbf{A}$ and $\mathbf{B}$ are expressed with each dot representing 1 sample. ${ }^{*} P<0.05 ;{ }^{* *} P<0.01$; ${ }^{* *} P<0.001 ;{ }^{* * *} P<0.0001$, versus tumor/saline group (A) or Neph/saline group (B), 1-way ANOVA.

early and frequent dosing via the s.c. route was the most effective method for treating cachexia. Oral administration is the most convenient and feasible route for clinical application. With this in mind, we performed intragastric gavage of TCMCBO7 of the animals of the acute LPS model in several experiments and found positive effects, suggesting its oral availability. However, we were not able to conduct the oral administration in the cancer and CKD cachexia models for several reasons. First, the gavage method is extremely difficult for sick and fragile animals, particularly at the last stage of cachexia. Second, unlike dosing in the acute model, chronic models require serial handling and restraint for the gavage, which represents a

Importantly, ТСMCB07 was detectable in the circulation and the concentrations corresponded to administered drug doses. Fourteen-day food intake and body weight gain were positively correlated with plasma ТСМСВ07 concentration, which supports the notion that s.c. administration of ТСМСВ07 stimulates appetite in a dose-dependent fashion. We did not find a significant difference in plasma BUN and Cr among all nephrectomy rats regardless of treatment, indicating that improvements in food intake, body weight, and lean mass were related to factors other than a change in renal function. Furthermore, 14-day food intake was not correlated with plasma BUN, but was negatively correlated with Cr. Plasma $\mathrm{Cr}$ is more reliable and accurate for reflecting renal function than BUN because plasma BUN is highly affected by extrarenal factors, such as heart failure, dehydration, liver function, or dietary protein $(57,58)$.

Route of delivery is a crucial factor that often determines an agent's efficacy and feasibility in a clinical setting. We initially used a central approach (i.c.v. administration) and tested to determine whether TCMCs have effective melanocortin antagonist properties. With 1 dose of i.c.v. injection, all 11 TCMCs showed a robust effect in stimulation of appetite. However, direct central delivery of this type of drugs is a barrier that would prevent clinical application. The capability of melanocortin antagonists crossing through the $\mathrm{BBB}$ is a substantial challenge for development of this class of drug. For example, AgRP and SHU9119 (melanocortin antagonists) or melanotan-II (melanocortin agonist) has no effects with peripheral administration, although these drugs are capable of inducing robust responses when given centrally $(44,59,60)$. Our data showed that ТСMCBO7 efficiently penetrated the BBB and effectively inhibited central melanocortin signaling. Furthermore, we observed that, with the same dose of TCMCBO7, initiation of chronic additional stress for the cachectic animals. The nonspecific stress would dramatically disrupt the rats' feeding behavior and increase their morbidity and mortality. Third, alternative methods of oral delivery (e.g., through drinking water or mixed with diet, etc.) can be undertaken, but it is difficult to ensure effective and consistent therapeutic dosing with these methods.

Because hypothalamic inflammation is an essential driver for both acute illness responses and cachexia $(61,62)$, we specifically analyzed inflammatory gene expression in hypothalamus to determine whether ТСМСBO7 s.c. treatment diminishes hypothalamic inflammation associated with cancer and CKD cachexia. The expression of Il1b, Illr1, and Il6 was suppressed by TCMCB07 treatment in cachexia associated with cancer. Determining whether this represents an intrinsic property of this compound or is simply reflective of the amelioration of end organ dysfunction (e.g., gut leak) secondary to catabolism will require further investigation. We note that our CKD model does not produce significant CNS inflammation, but this is correlated with the relatively mild (albeit prolonged) cachexia typical of this model. Furthermore, it is possible that the cachexia in this model is at least partially driven by relative hyperleptinemia, and this would also be expected to respond to melanocortin antagonism $(30,63)$. The Selp gene encoding P selectin is associated with the development of cachexia in tumor-bearing rats, LPS-treated mice, and patients with cancer $(46,64)$. Consistent with these, we found a marked increase of Selp gene expression in hypothalamus among all tumor-bearing rats relative to sham-treated rats. Interestingly, s.c. TCMCB07 treatment dramatically suppressed Selp gene expression in tumor-bearing rats compared with saline treatment, suggesting that Selp gene expression is a sensitive predicator for cancer cachexia and that ТСМСВ07 treatment effectively inhibits inflammation 
during cancer progression and cachexia development. Furthermore, previous studies demonstrated that both acute and chronic inflammation decrease hypothalamic Pomc transcription and AgRP secretion, while simultaneously increasing Agrp transcription and $\alpha$-MSH secretion $(20,22)$. The alteration in hypothalamic Pomc and Agrp gene expression was also observed in a unique setting of severe muscle catabolism associated with essential amino acid deficiency (65). Consistent with these findings, we observed a significant $(P<$ 0.001) upregulation of hypothalamic Agrp transcription in both cancer- and CKD-associated cachexia and a trend toward reduced Pomc transcription in cancer, but not CKD cachexia. Notably, TCMCBO7 s.c. treatment remarkably suppressed Agrp transcription in both cancer- and CKD-associated cachexia, suggesting that endogenous Agrp transcription remained sensitive to overall body weight and food intake status. Obviously, there are many other factors regulating food intake that were not explored in this study and that therefore deserve further study in the future, including the expression of peripheral factors (e.g., ghrelin) and various ligands and receptors in the CNS (e.g., growth hormone secretagogue receptor 1 , neuropeptide $\mathrm{Y}$, etc.).

Despite tremendous progress in understanding the mechanisms of cachexia, therapeutic interventions for this common condition associated with many advanced illnesses are lacking. Because cachexia is driven by a variable combination of reduced food intake, increased energy expenditure, excess catabolism, and inflammation (5), a single drug therapy is unlikely to be sufficient to treat this condition. Instead, it will likely require optimized therapeutic combinations with effective orexigenic, antiinflammatory, and anticatabolic agents $(66,67)$. TCMCB07, a synthetic orexigenic agent, was developed through a classical approach against central melanocortin signaling, and it showed high efficacy in attenuation of anorexia, body weight loss, fat mass loss, and muscle wasting associated with cachexia. This preclinical trial demonstrates that TCMCB07 is a promising drug candidate for cachexia therapy. We anticipate that combination therapy with TMCMBO7 and additional drugs that target overactivation of catabolic processes and inflammation will greatly benefit patients with cachexia.

\section{Methods}

Animals. Sprague-Dawley (SD) and F344/CDF (F344) male rats (Charles River) weighing 225-275 g were housed at 2 per cage, fed rat chow (Diet 5001; Purina Mills, Inc.), and acclimated for at least 7 days before use. SD strain rats were used for LPS and CKD models, and F344 was used for the tumor model. One day before each experiment commenced, animals were weighed and divided into treatment groups so that the mean body weights of each group were similar. During experiments, food intake and body weight were measured at the same time of each day, unless otherwise noted.

Compounds. Eleven TCMCs (TCMCB01-10 plus TCMCB07A) were synthetic MC4R antagonists that were designed and provided by Tensive Controls Inc. Each compound was dissolved in distilled water by vortex or sonication for each experiment, and fresh working solution was prepared before administration. Tests of each TCMC began with pilot experiments for selecting doses via 4 administration routes (i.c.v., i.p., s.c., oral). Among 11 TCMCs, TCMCB07 was finally chosen for comprehensive evaluation based on the results from a series of pilot studies. Melanocortin antagonists AgRP and SHU9119 (Phoenix Pharmaceuticals) were used as positive control reagents but were only effective with central administration. PG932 (provided by Tensive Controls Inc.), a synthetic derivative of SHU9119, was previously reported as an effective reagent via i.p. injection in an LPS mouse model (42) that was also used as a potential positive control for peripheral treatment.

Compound administration. For central routes and doses, we first investigated the effects on stimulation of appetite and attenuation of body weight loss after central administration of TCMCs. To establish a route for central drug administration, unilateral cannulation of the lateral ventricle was performed. Under isoflurane anesthesia, 22-gauge lateral ventricle cannulas (Plastics One) were placed in rats using a stereotactic instrument (Kopf) at the following coordinates relative to bregma: $1.5 \mathrm{~mm}$ (X), $-1.0 \mathrm{~mm}(\mathrm{Y})$, and $-4.0 \mathrm{~mm}(\mathrm{Z})$. Rats were then individually housed and allowed to recover from surgery for at least 7 days. Compounds at $2 \mu \mathrm{g} / \mathrm{rat} / \mathrm{d}(1.5 \mathrm{nmol} / \mathrm{rat} / \mathrm{d})$ or saline and positive control agents were administered in a total volume of $5 \mu \mathrm{L}$ via i.c.v. injection. For peripheral routes and doses, 3 routes were applied for peripheral administration: i.p., s.c. injection, and oral intragastric gavage. The dose range of TCMCs was $0.6-12 \mathrm{mg} / \mathrm{kg} / \mathrm{d}$. Dosing frequency was between once and twice daily.

Acute study in LPS-induced anorexia and body weight loss. The effects of each TCMC in the attenuation of anorexia and body weight loss were first examined in LPS-treated rats. LPS doses were selected through a preliminary dose-response experiment. LPS (MilliporeSigma) was dissolved in vehicle (0.5\% BSA in 0.9\% saline) and injected into SD rats via i.p. injection at doses of 0 (vehicle), 10, 50, 100, and $250 \mu \mathrm{g} / \mathrm{kg} / \mathrm{d}$, respectively. Under fasting conditions, body weight change at 24 hours after LPS injection was measured and muscle catabolism was analyzed by quantitative reverse-transcriptase PCR (qRT-PCR). An LPS dose of 100-250 $\mu \mathrm{g} / \mathrm{kg} / \mathrm{d}$ was chosen for the compound test. In order to accurately measure spontaneous food intake during acute response to LPS and compounds, we coordinated with rat nocturnal behavior and performed a series of night-feeding studies in a very consistent way $(29,65)$. Briefly, rats were individually housed for at least 7 days for acclimation before starting experiments. One day before treatment, rats were weighed and placed in clean cages, and preweighed food pellets were placed into each cage at 2:30 pm for measuring baseline of 24 hours of food intake and body weight change. On the day of treatment at 2:30 pm, rats were weighed for initial body weight and the remaining food was weighed and removed from cages. LPS i.p. injection was performed at $3 \mathrm{pm}$, and at $4 \mathrm{pm}$, saline or compound administration was performed through i.c.v. injection $(1.5 \mathrm{nmol} / \mathrm{rat})$, i.p. injection $(3 \mathrm{mg} / \mathrm{kg} / \mathrm{d})$, and intragastric gavage $(10 \mathrm{mg} / \mathrm{kg} / \mathrm{d})$. Preweighed food pellets were placed into each cage at 5:30 pm, and then food weights were measured at 2 hours, 4 hours (under red light illumination during night phase), 16 hours, 24 hours, and 48 hours after food was returned. Body weights were measured at 16 hours, 24 hours, and 48 hours. Care was taken to minimize nonspecific stress to the animals during nighttime food measurements.

Cancer cachexia model. The cancer cachexia model was generated in F344 rats. Our previous studies and others' demonstrate that the rat MCA sarcoma model produces reliable and reproducible cancer cachexia; and the MCA tumor is not rejected by F344 rat strain, nor does it metastasize $(34,68)$. Based on our experience with this model for these experiments, we modified it by performing tumor implantation 6 to 10 days before treatment to allow for adequate tumor growth. Briefly, under isoflurane anesthesia, frozen tumor tissue was implanted s.c. into the flanks of donors for generating fresh tumor tissue. Approximately 16 days later, fresh tumor tissue (1.0-1.2 g) from a euthanized 
donor was implanted s.c. into the flanks of a rat under isoflurane anesthesia. Sham-operated rats received the procedure without tumor tissue and served as experimental controls. Rats were administered postoperatively with analgesic (buprenorphine $0.05 \mathrm{mg} / \mathrm{kg}$, s.c.) and then individually housed. Daily body weight and food intake were measured. Tumors became palpable 6 to 7 days after implantation, and tumor size was measured daily thereafter. Tumor volume was calculated from the formula for a prolate sphere: $V=1 / 2 a b^{2}$, where $a$ is the longer and $b$ the shorter dimension (69). On days 12 to 14 , animals were euthanized, at the point when tumor growth and overall conditions of tumor-bearing animals had fallen within predetermined endpoints of the study, with particular attention paid to the volume of tumor and overall health.

CKD-associated cachexia model. Two-stage 5/6 nephrectomy surgery was performed in SD rats for a CKD-associated cachexia model, and sham controls experienced the same procedures without excision of kidney tissue, as described previously (33). Briefly, for the stage I surgery (Neph-I), the animals were anesthetized with isoflurane and placed prone in a clean environment. $\mathrm{A} 1 \mathrm{~cm}$ posterior incision was made on the left flank through which the left kidney was located. For animals undergoing nephrectomy, the renal capsule was removed, the upper and lower one-third of the kidney was transected, and the resultant wound was cauterized, leaving the middle one-third of the kidney with renal artery and vein intact. For control animals receiving a sham operation, the renal capsule was opened up to simulate the manipulations performed in the nephrectomy. The surgical wounds were then closed via suture at the muscle and skin layers. Animals that received surgery were allowed to recover and individually housed. Seven days after stage I surgery, animals were again anesthetized and placed prone in the surgical area for the stage II surgery (Neph-II). This time, a right $1 \mathrm{~cm}$ incision was performed and the right kidney was isolated. For animals undergoing nephrectomy, the renal capsule was removed and vasculature was tied off with suture. The vascular bundle was then transected distal to the suture, and the entire kidney was removed. For animals in the sham-treatment group, the renal capsule was removed. The surgical wounds were closed with suture at the muscle and skin layers. A dose of analgesic was administered after each stage of surgery (buprenorphine, $0.05 \mathrm{mg} / \mathrm{kg}$, s.c.).

Long-term studies with cachexia models of cancer and CKD. To determine the effects of TCMCBO7 treatment in cachexia associated with cancer and CKD, we designed different experimental time frames for performing surgeries, measurements, treatments, and tissue collections (Figure 2A, Figure 3A, and Figure 5A). Tumor implantation and 2-stage 5/6 nephrectomy or sham operation were performed at the beginning of each experiment. Food intake and body weight were measured at a similar time point of each day, and body composition was measured before and after compound administration. In the study with cancer cachexia, when symptoms, such as anorexia and lethargy, appeared in tumor-bearing animals, saline or compound was administered through i.c.v. or i.p. injection once a day and s.c. injection twice a day for a total of 4 to 6 days. In the study with CKD cachexia, starting at day 14 (14 days after Neph-I), saline or compound was administered via s.c. injection twice a day for a total of 14 days.

Body composition. Body fat mass and lean mass were determined before and after administration of compounds using magnetic resonance relaxometry (EchoMRI 4-in-1 Live Animal Composition Analyzer; Echo Medical System).
Blood and tissue collection. At the end of experiments with cancer and CKD cachexia, rats were euthanized. Blood was collected at approximately 2 hours after the last injection of TCMCBO7 (estimated $\mathrm{C}_{\max }$ ) through cardiac puncture, and plasma was isolated and stored at $-80^{\circ} \mathrm{C}$ until analysis. The brains were dissected, snap-frozen, and then stored at $-80^{\circ} \mathrm{C}$ until analysis. Tumors from tumor-bearing animals were dissected away from surrounding tissue and weighed. The residual kidneys in nephrectomized animals were examined for final confirmation of the procedure and residual renal survival.

Plasma TCMCBO7, BUN and Cr assay, and brain tissue qRT-PCR analysis. We developed an assay for TCMCBO7 in body fluids using reversephase HPLC with aromatic amino acid fluorescence detection. This method used a Hypersil GOLD C- 18 column ( $4.6 \mathrm{~mm}$ ID/25 cm length, 5 $\mu \mathrm{m}$ particle size, $17.5 \mathrm{~nm}$ pore size), with a $15 \%-50 \%$ acetonitrile/0.01\% hydrochloric acid gradient. We used the unique fluorescence spectrum of these peptide's naphthylalanine (Nal) residue (229 excitation and 337 emission, nanometer) for postcolumn detection of eluting Nal-containing peptides with a Fluoat-01 Panorama Spectrofluorometer. Plasma extraction was with acetonitrile with $0.01 \%$ hydrochloric acid. This 1-step approach precipitated plasma proteins, while essentially extracting $100 \%$ of the drug. Standard curves were generated by spiking blank plasma samples with known peptide amounts. Plasma samples were assayed for concentration of BUN and $\mathrm{Cr}$ with a biochemistry analyzer (Siemens Dimension Vista 1500 Chemistry Analyzer). Total RNA was extracted from hypothalamic blocks using a QIAGEN RNA Mini Kit, and gene expression was analyzed by qRT-PCR as described previously (64).

Statistics. All data are expressed as mean \pm SEM for each group. Statistical analyses were performed using unpaired 2-tailed Student's $t$ test and 1-way ANOVA or 2-way ANOVA analysis followed by Bonferroni's posttests using GraphPad Prism 8. The correlation was analyzed using Pearson correlation coefficient and linear regression. $P<0.05$ was considered statistically significant.

Study approval. Studies were approved by the IACUC of the Oregon Health and Science University and conducted according to the NIH Guide for the Care and Use of Laboratory Animals (National Academies Press, 2011).

\section{Author contributions}

$\mathrm{XZ}$ and DLM designed the research studies. XZ, MFC, and MS conducted experiments. KAG designed all of the TCMCs. XZ and DLM analyzed data. XZ wrote the manuscript. DLM and MFC reviewed and edited the manuscript. All authors approved the final version of the manuscript.

\section{Acknowledgments}

We thank My Linh Nguyen and Tariq Shah for technical assistance. This work was supported by NCI R01 CA184324 (to DLM); NCI 1R43CA150703, 2R44CA150703, and 2R44CA210763 (to KAG); and a research grant from Tensive Controls Inc.

Address correspondence to: Daniel L. Marks, Papé Family Pediatric Research Institute and Knight Cancer Institute, Oregon Health and Science University, 3181 SW Sam Jackson Park Road, L 481, Portland, Oregon 97239, USA. Phone: 503.494.6218; Email: marksd@ohsu.edu. 
1. Mak RH, Ikizler AT, Kovesdy CP, Raj DS, Stenvinkel P, Kalantar-Zadeh K. Wasting in chronic kidney disease. J Cachexia Sarcopenia Muscle. 2011;2(1):9-25.

2. Tisdale MJ. Biology of cachexia. J Natl Cancer Inst. 1997;89(23):1763-1773.

3. Larkin M. Thwarting the dwindling progression of cachexia. Lancet. 1998;351(9112):1336.

4. Argilés JM, Busquets S, Stemmler B, López-Soriano FJ. Cancer cachexia: understanding the molecular basis. Nat Rev Cancer. 2014;14(11):754-762.

5. Baracos VE, Martin L, Korc M, Guttridge DC, Fearon KCH. Cancer-associated cachexia. Nat Rev Dis Primers. 2018;4:17105.

6. Fearon KC, Glass DJ, Guttridge DC. Cancer cachexia: mediators, signaling, and metabolic pathways. Cell Metab. 2012;16(2):153-166.

7. Mak RH, Cheung WW. Is ghrelin a biomarker for mortality in end-stage renal disease? Kidney Int. 2011;79(7):697-699.

8. Cone RD. Anatomy and regulation of the central melanocortin system. Nat Neurosci. 2005;8(5):571-578.

9. Garfield AS, Lam DD, Marston OJ, Przydzial MJ, Heisler LK. Role of central melanocortin pathways in energy homeostasis. Trends Endocrinol Metab. 2009;20(5):203-215.

10. Nuzzaci D, et al. Plasticity of the melanocortin system: determinants and possible consequences on food intake. Front Endocrinol (Lausanne). 2015;6:143.

11. Baldini G, Phelan KD. The melanocortin pathway and control of appetite-progress and therapeutic implications. JEndocrinol. 2019;241(1):R1-R33.

12. Dietrich MO, Liu ZW, Horvath TL. Mitochondrial dynamics controlled by mitofusins regulate Agrp neuronal activity and diet-induced obesity. Cell. 2013;155(1):188-199.

13. Girardet C, Butler AA. Neural melanocortin receptors in obesity and related metabolic disorders. Biochim Biophys Acta. 2014;1842(3):482-494.

14. Hill JW, Faulkner LD. The role of the melanocortin system in metabolic disease: new developments and advances. Neuroendocrinology. 2017;104(4):330-346.

15. Burfeind KG, Michaelis KA, Marks DL. The central role of hypothalamic inflammation in the acute illness response and cachexia. Semin Cell Dev Biol. 2016;54:42-52.

16. Arruda AP, et al. Hypothalamic actions of tumor necrosis factor alpha provide the thermogenic core for the wastage syndrome in cachexia. Endocrinology. 2010;151(2):683-694.

17. Grossberg AJ, et al. Inflammation-induced lethargy is mediated by suppression of orexin neuron activity. J Neurosci. 2011;31(31):11376-11386.

18. Marks DL, Ling N, Cone RD. Role of the central melanocortin system in cachexia. Cancer Res. 2001;61(4):1432-1438.

19. Braun TP, et al. Expression of myeloid differentiation factor 88 in neurons is not requisite for the induction of sickness behavior by interleukin-1 $\beta$. J Neuroinflammation. 2012;9:229.

20. Grossberg AJ, et al. Arcuate nucleus proopiomelanocortin neurons mediate the acute anorectic actions of leukemia inhibitory factor via gp130. Endocrinology. 2010;151(2):606-616.
21. Scarlett JM, et al. Regulation of central melanocortin signaling by interleukin-1 beta. Endocrinology. 2007;148(9):4217-4225.

22. Scarlett JM, et al. Regulation of agouti-related protein messenger ribonucleic acid transcription and peptide secretion by acute and chronic inflammation. Endocrinology. 2008;149(10):4837-4845.

23. Argilés JM, Moore-Carrasco R, Fuster G, Busquets S, López-Soriano FJ. Cancer cachexia: the molecular mechanisms. Int J Biochem Cell Biol. 2003;35(4):405-409.

24. Burfeind KG, Zhu X, Levasseur PR, Michaelis KA, Norgard MA, Marks DL. TRIF is a key inflammatory mediator of acute sickness behavior and cancer cachexia. Brain Behav Immun . 2018;73:364-374.

25. Burfeind KG, et al. Microglia in the hypothalamus respond to tumor-derived factors and are protective against cachexia during pancreatic cancer. Glia. 2020;68(7):1479-1494.

26. Cheung WW, Paik KH, Mak RH. Inflammation and cachexia in chronic kidney disease. Pediatr Nephrol. 2010;25(4):711-724.

27. Michaelis KA, et al. The TLR7/8 agonist R848 remodels tumor and host responses to promote survival in pancreatic cancer. Nat Commun. 2019;10(1):4682.

28. Zhu X, et al. MyD88 signalling is critical in the development of pancreatic cancer cachexia. JCachexia Sarcopenia Muscle. 2019;10(2):378-390.

29. Zhu X, Levasseur PR, Michaelis KA, Burfeind KG, Marks DL. A distinct brain pathway links viral RNA exposure to sickness behavior. Sci Rep. 2016;6:29885.

30. Cheung W, Yu PX, Little BM, Cone RD, Marks DL, Mak RH. Role of leptin and melanocortin signaling in uremia-associated cachexia. J Clin Invest. 2005;115(6):1659-1665.

31. Cheung WW, et al. A pegylated leptin antagonist ameliorates CKD-associated cachexia in mice. J Am Soc Nephrol. 2014;25(1):119-128.

32. Currow DC, Maddocks M, Cella D, Muscaritoli M. Efficacy of anamorelin, a novel non-peptide ghrelin analogue, in patients with advanced non-small cell lung cancer (NSCLC) and cachexia-review and expert opinion. Int J Mol Sci. 2018;19(11):E3471.

33. Deboer MD, et al. Ghrelin treatment of chronic kidney disease: improvements in lean body mass and cytokine profile. Endocrinology. 2008;149(2):827-835.

34. DeBoer MD, et al. Ghrelin treatment causes increased food intake and retention of lean body mass in a rat model of cancer cachexia. Endocrinology. 2007;148(6):3004-3012.

35. Nishie K, Yamamoto S, Nagata C, Koizumi T, Hanaoka M. Anamorelin for advanced nonsmall-cell lung cancer with cachexia: Systematic review and meta-analysis. Lung Cancer. 2017;112:25-34

36. Temel JS, et al. Anamorelin in patients with nonsmall-cell lung cancer and cachexia (ROMANA 1 and ROMANA 2): results from two randomised, double-blind, phase 3 trials. Lancet Oncol. 2016;17(4):519-531.

37. DeBoer MD. Update on melanocortin interventions for cachexia: progress toward clinical application. Nutrition. 2010;26(2):146-151.
38. DeBoer MD, Marks DL. Therapy insight: Use of melanocortin antagonists in the treatment of cachexia in chronic disease. Nat Clin Pract Endocrinol Metab. 2006;2(8):459-466.

39. Scarlett JM, Marks DL. The use of melanocortin antagonists in cachexia of chronic disease. Expert Opin Investig Drugs. 2005;14(10):1233-1239.

40. Dallmann R, et al. The orally active melanocortin-4 receptor antagonist BL-6020/979: a promising candidate for the treatment of cancer cachexia. J Cachexia Sarcopenia Muscle. 2011;2(3):163-174.

41. Markison S, et al. The regulation of feeding and metabolic rate and the prevention of murine cancer cachexia with a small-molecule melanocortin-4 receptor antagonist. Endocrinology. 2005;146(6):2766-2773.

42. Sutton GM, Josephine Babin M, Gu X, Hruby VJ, Butler AA. A derivative of the melanocortin receptor antagonist SHU9119 (PG932) increases food intake when administered peripherally. Peptides. 2008;29(1):104-111.

43. Bison S, Carboni L, Arban R, Bate S, Gerrard P, Razzoli M. Differential behavioral, physiological, and hormonal sensitivity to LPS challenge in rats. Int J Interferon Cytokine Mediat Res. 2009;1:1-13.

44. Wisse BE, Frayo RS, Schwartz MW, Cummings DE. Reversal of cancer anorexia by blockade of central melanocortin receptors in rats. Endocrinology. 2001;142(8):3292-3301.

45. Ramos EJ, et al. Effects of omega-3 fatty acid supplementation on tumor-bearing rats. J Am Coll Surg. 2004;199(5):716-723.

46. Tan BH, et al. P-selectin genotype is associated with the development of cancer cachexia. $E M B O$ Mol Med.2012;4(6):462-471.

47. Scarlett JM, Bowe DD, Zhu X, Batra AK, Grant WF, Marks DL. Genetic and pharmacologic blockade of central melanocortin signaling attenuates cardiac cachexia in rodent models of heart failure. JEndocrinol. 2010;206(1):121-130.

48. Catania A, Lonati C, Sordi A, Carlin A, Leonardi P, Gatti S. The melanocortin system in control of inflammation. ScientificWorldJournal. 2010;10:1840-1853.

49. Wang W, Guo DY, Lin YJ, Tao YX. Melanocortin regulation of inflammation. Front Endocrinol (Lausanne). 2019;10:683.

50. Alves MJ, et al. Adipose tissue fibrosis in human cancer cachexia: the role of TGF $\beta$ pathway. BMC Cancer. 2017;17(1):190.

51. Uomo G, Gallucci F, Rabitti PG. Anorexia-cachexia syndrome in pancreatic cancer: recent development in research and management. JOP. 2006;7(2):157-162.

52. Jatoi A, et al. Dronabinol versus megestrol acetate versus combination therapy for cancer-associated anorexia: a North Central Cancer Treatment Group study. JClin Oncol. 2002;20(2):567-573.

53. Simons JP, Schols AM, Hoefnagels JM, Westerterp KR, ten Velde GP, Wouters EF. Effects of medroxyprogesterone acetate on food intake, body composition, and resting energy expenditure in patients with advanced, nonhormonesensitive cancer: a randomized, placebo-controlled trial. Cancer. 1998;82(3):553-560.

54. Grossberg AJ, et al. Association of body composition with survival and locoregional control of 


\section{RESEARCH ARTICLE}

radiotherapy-treated head and neck squamous cell carcinoma. JAMA Oncol. 2016;2(6):782-789.

55. Fouladiun M, Körner U, Bosaeus I, Daneryd P, Hyltander A, Lundholm KG. Body composition and time course changes in regional distribution of fat and lean tissue in unselected cancer patients on palliative care--correlations with food intake, metabolism, exercise capacity, and hormones. Cancer. 2005;103(10):2189-2198.

56. Barazzoni R, et al. Combined effects of ghrelin and higher food intake enhance skeletal muscle mitochondrial oxidative capacity and AKT phosphorylation in rats with chronic kidney disease. Kidney Int. 2010;77(1):23-28.

57. Lyman JL. Blood urea nitrogen and creatinine. Emerg Med Clin North Am. 1986;4(2):223-233.

58. Walker HK HW, Hurst JW, eds. Clinical Methods: The History, Physical, and Laboratory Examinations. Butterworths; 1990.

59. Cheung WW, Mak RH. Melanocortin antagonism ameliorates muscle wasting and inflammation in chronic kidney disease. Am J Physiol Renal Physiol. 2012;303(9):F1315-F1324.

60. Fan W, Boston BA, Kesterson RA, Hruby VJ, Cone $\mathrm{RD}$. Role of melanocortinergic neurons in feeding and the agouti obesity syndrome. Nature. 1997;385(6612):165-168.

61. Dwarkasing JT, Marks DL, Witkamp RF, van Norren K. Hypothalamic inflammation and food intake regulation during chronic illness. Peptides. 2016;77:60-66.

62. Grossberg AJ, Scarlett JM, Marks DL. Hypothalamic mechanisms in cachexia. Physiol Behav. 2010;100(5):478-489.

63. Mak RH, Cheung W, Cone RD, Marks DL. Mechanisms of disease: Cytokine and adipokine signaling in uremic cachexia. Nat Clin Pract Nephrol. 2006;2(9):527-534.

64. Braun TP, et al. Central nervous system inflammation induces muscle atrophy via activation of the hypothalamic-pituitary-adrenal axis. J Exp Med. 2011;208(12):2449-2463.
65. Zhu X, et al. Hypothalamic signaling in anorexia induced by indispensable amino acid deficiency. Am J Physiol Endocrinol Metab. 2012;303(12):E1446-E1458.

66. Advani SM, Advani PG, VonVille HM, Jafri SH. Pharmacological management of cachexia in adult cancer patients: a systematic review of clinical trials. BMC Cancer. 2018;18(1):1174.

67. Marceca GP, Londhe P, Calore F. Management of cancer cachexia: attempting to develop new pharmacological agents for new effective therapeutic options. Front Oncol. 2020;10:298.

68. Sato T, Meguid MM, Fetissov SO, Chen C, Zhang L. Hypothalamic dopaminergic receptor expressions in anorexia of tumor-bearing rats. Am J Physiol Regul Integr Comp Physiol. 2001;281(6):R1907-R1916.

69. Faustino-Rocha A, et al. Estimation of rat mammary tumor volume using caliper and ultrasonography measurements. Lab Anim (NY). 2013;42(6):217-224. 